\title{
Production of dark-matter bound states in the early universe by three-body recombination
}

\author{
Eric Braaten, ${ }^{a}$ Daekyoung $\mathrm{Kang}^{b}$ and Ranjan Laha ${ }^{c}$ \\ ${ }^{a}$ Department of Physics, The Ohio State University, \\ Columbus, OH 43210, U.S.A. \\ ${ }^{b}$ Key Laboratory of Nuclear Physics and Ion-beam Application (MOE) and \\ Institute of Modern Physics, Fudan University, \\ Shanghai 200433, China \\ ${ }^{c}$ PRISMA Cluster of Excellence and Mainz Institute for Theoretical Physics, \\ Johannes Gutenberg-Universität Mainz, \\ 55099 Mainz, Germany \\ E-mail: braaten.1@osu.edu, dkang@fudan.edu.cn, ranjalah@uni-mainz.de
}

ABSTRACT: The small-scale structure problems of the universe can be solved by selfinteracting dark matter that becomes strongly interacting at low energy. A particularly predictive model for the self-interactions is resonant short-range interactions with an Swave scattering length that is much larger than the range. The velocity dependence of the cross section in such a model provides an excellent fit to self-interaction cross sections inferred from dark-matter halos of galaxies and clusters of galaxies if the dark-matter mass is about $19 \mathrm{GeV}$ and the scattering length is about $17 \mathrm{fm}$. Such a model makes definite predictions for the few-body physics of weakly bound clusters of the dark-matter particles. The formation of the two-body bound cluster is a bottleneck for the formation of larger bound clusters. We calculate the production of two-body bound clusters by three-body recombination in the early universe under the assumption that the dark matter particles are identical bosons, which is the most favorable case. If the dark-matter mass is $19 \mathrm{GeV}$ and the scattering length is $17 \mathrm{fm}$, the fraction of dark matter in the form of two-body bound clusters can increase by as much as 4 orders of magnitude when the dark-matter temperature falls below the binding energy, but its present value remains less than $10^{-6}$. The present fraction can be increased to as large as $10^{-3}$ by relaxing the constraints from small-scale structure and decreasing the mass of the dark matter particle.

Keywords: Beyond Standard Model, Cosmology of Theories beyond the SM

ArXiv ePrint: 1806.00609 


\section{Contents}

1 Introduction 1

2 Universal two-body physics with large scattering length 4

2.1 Two-body physics 4

2.2 Dark matter parameters 6

3 Universal three-body physics of identical bosons $\quad 8$

3.1 Trimer spectrum 8

$\begin{array}{llr}3.2 & \text { Dimer-atom scattering } & 9\end{array}$

$\begin{array}{llr}3.3 & \text { Three-body recombination } & 12\end{array}$

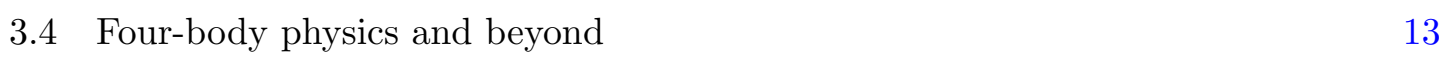

4 Rate coefficients at thermal equilibrium $\quad \mathbf{1 3}$

$\begin{array}{ll}4.1 \text { Inelastic atom-atom scattering } & 13\end{array}$

$\begin{array}{lll}4.2 & \text { Dimer breakup } & 14\end{array}$

$\begin{array}{lll}4.3 & \text { Three-body recombination } & 16\end{array}$

5 Early universe $\quad 17$

$\begin{array}{lll}5.1 & \text { Rate equations } & 18\end{array}$

5.2 Approximation in scaling and threshold regions 20

$\begin{array}{lll}5.3 & \text { Numerical results } & 22\end{array}$

6 Discussion and conclusion $\quad 24$

\section{Introduction}

The simplest paradigm for dark matter is that it consists of weakly interacting elementary particles. However visible matter consists not only of elementary particles, such as electrons, but also of composite particles, such as nuclei. Nuclei are clusters of protons and neutrons bound by residual forces from QCD. Protons and neutrons consist of quarks bound by the color force of QCD. Dark matter could also consist of composite particles. In particular, it could consist of "dark nucleons" and bound clusters of dark nucleons ("dark nuclei"). The dark nucleons could be elementary or composite, but they have an integer-valued conserved charge that we call "dark baryon number". Various models for dark-matter bound states have been discussed in the literature, including a near-threshold S-wave resonance [1-3], the exchange of a light mediating boson between elementary fermions, QCD-like structure in the dark sector, and other mechanisms [4-34]. Light nuclei up to ${ }^{7} \mathrm{Li}$ are produced in the early universe by big-bang nucleosynthesis $[35,36]$. The relevant reactions are all 2body collisions of nuclei. Some of the 2-body reactions that produce a nucleus with larger baryon number than either of the colliding nuclei are rearrangement reactions, such as 
$d+d \rightarrow{ }^{3} \mathrm{He}+n$. However the most important such reactions are radiative fusion reactions, such as $p+d \rightarrow{ }^{3} \mathrm{He}+\gamma$, in which the two incoming nuclei coalesce while radiating a photon to conserve energy and momentum. The effects of 3-body collisions are negligible in big-bang nucleosynthesis. Three-body collisions do play a role in stellar nucleosynthesis despite the relatively low density. In particular, the Hoyle reaction $\alpha+\alpha+\alpha \rightarrow{ }^{12} \mathrm{C}+\gamma$ provides a pathway around the bottleneck caused by the relatively large binding energy of the ${ }^{4} \mathrm{He}$ nucleus $\alpha$.

If dark matter consists of dark nucleons that can form bound clusters, these dark nuclei can be produced in the early universe by "dark nucleosynthesis". Studies have shown that a sequence of dark nuclei with increasing dark baryon number can indeed be produced in the early universe $[9,11,14,37]$. The relevant few-body mechanisms were assumed to be 2-body radiative fusion reactions, in which two incoming dark nuclei coalesce while radiating a much lighter particle to conserve energy and momentum. If there is no such light particle, dark nuclei with larger dark baryon numbers must instead be built up through rearrangement collisions. If dark nuclei with dark baryon number 2 ("dark deuterons") have already been formed, dark nuclei with larger dark baryon numbers can be produced by rearrangement collisions of two dark nuclei, in which dark nucleons are transferred between the two colliding nuclei. However the production of the dark deuterons is a bottleneck that can only be overcome by collisions of 3 or more dark nucleons. The simplest such reaction is the 3-body recombination of three dark nucleons into a dark deuteron and a recoiling dark nucleon. Whether a significant population of dark deuterons can be produced in the early universe can only be determined by detailed calculations using specific models for the few-body physics.

One class of models for few-body physics that are extremely predictive are those with short-range interactions and an S-wave resonance very close to the scattering threshold for a pair of particles [38]. In these models, the elastic scattering cross section for a pair of particles has dramatic energy dependence that is completely determined by the particle mass $m$ and the S-wave scattering length $a$, which is much larger that the range $r_{0}$ of the interactions. When the center-of-mass collision energy $E$ decreases below the energy scale $1 / m r_{0}^{2}$ set by the range, the elastic cross section increases as $1 / E$, nearly saturating the Swave unitarity bound. The cross section levels off when $E$ decreases below the energy scale $1 / m a^{2}$, approaching a large value proportional to $a^{2}$ as $E$ approaches 0 . If $a$ is positive, the S-wave resonance is a stable bound cluster. This weakly bound cluster is universal, in the sense that it has properties determined by $a$, including a small binding energy $1 / m a^{2}$ and large geometric size of order $a$. The universality in the two-particle sector for particles with a large scattering length extends to the 3 -particle and higher sectors, although it can be more intricate [38]. It strongly constrains the universal bound clusters, whose binding energies are smaller than the energy scale set by the range. Whether there are universal bound clusters with 3 or more particles depends on the symmetries of the particles. The simplest case in which there are such universal bound clusters is identical spin-0 bosons. Universality also provides strong constraints on reaction rates in the 3-particle and higher sectors [38]. These reaction rates can display dramatic resonant enhancements at low energy. For example, the 3 -body recombination rate can increase as $1 / E^{2}$ when the center- 
of-mass collision energy $E$ decreases below the energy scale $1 / m r_{0}^{2}$, and it can approach a large value proportional to $a^{4}$ in the low-energy limit.

Large low-energy cross sections for dark matter particles are motivated by discrepancies between observations of the small-scale structure of the universe and simulations based on collisionless cold dark matter [39-44]. Observations of dwarf galaxies are inconsistent with the cusp of dark matter at the center of a galaxy that is predicted by dark-matteronly simulations. Dark-matter-only simulations also imply that dwarf galaxies bound to the Milky Way should be denser than those that have been observed. Although other explanations for these problems have been proposed, they can all be solved by self-interacting dark matter that is strongly interacting at low energy $[45,46]$. Short-range interactions with a large scattering length provide a particularly predictive model of self-interactions that become strong at low energy [1-3].

If dark nucleons have short-range interactions with a large scattering length $a$, they have universal low-energy properties determined by $a$ [1]. We denote the dark nucleon by $d$ and a bound cluster of $n$ dark nucleons by $d_{n}$. If $a$ is negative, there are no weakly bound clusters of two dark nucleons. If $a$ is positive, there is one universal weakly bound cluster: the dark deuteron $d_{2}$. If a pair of dark nucleons has annihilation channels, the scattering length $a$ is complex with a small negative imaginary part. In addition to the elastic cross section and the binding energy of the dark deuteron, the annihilation rate of a pair of dark nucleons and the decay rate of the dark deuteron are also universal in the sense that they are determined by the complex scattering length [1]. In a direct detection experiment, the dark deuteron can scatter elastically from a target nucleus, or it can be broken up by the collision $[2,3,47]$. The low-energy cross sections for both processes are determined by $a$ up to a multiplicative factor. Their dependence on the collision energy and the recoil angle provides interesting signatures for this simplest dark nucleus. The simplest reaction that can form a bound cluster is 3-body recombination: $d+d+d \rightarrow d_{2}+d$. In an expanding and cooling thermal system, such as the early universe, the decreasing number density will tend to suppress 3-body recombination while the decreasing temperature will tend to enhance it. Once dark deuterons are produced, the competing breakup reaction $d_{2}+d \rightarrow d+d+d$ will destroy them. The net effect on the population of $d_{2}$ can only be determined by explicit calculations.

In this paper, we study 3-body recombination into dark deuterons during the Hubble expansion in the early universe under the assumption that the dark matter consists of dark nucleons that are identical bosons with a large positive scattering length, which is the most favorable case for the formation of universal bound clusters. We determine the mass $m_{\chi}$ and the scattering length $a$ of the dark nucleon that would be required to solve the small-scale structure problems of the universe. For these values of $m_{\chi}$ and $a$, the fraction of dark matter in the form of dark deuterons can increase by orders of magnitude when the dark-matter temperature decreases to below the binding energy of the dark deuteron. However, we find that a significant population of dark deuterons cannot be produced during the Hubble expansion. Since the production of the dark deuteron is a bottleneck, larger dark nuclei will also not be formed. A much larger population of dark deuterons can be produced if the constraints from small-scale structure are relaxed and the mass of the dark matter particle is decreased. 
In section 2, we summarize the universal 2-body physics of particles with a large scattering length. We also determine the mass and the scattering length of the dark nucleon that would be required to solve small-scale structure problems of the universe. In section 3 , we summarize the universal 3-body physics of identical bosons with large scattering length. In section 4, we present results for the rate constants for many-body systems of identical bosons with large scattering length in thermal equilibrium. In section 5 , we consider the formation of dark deuterons by 3-body recombination during the Hubble expansion of the early universe. We calculate the fraction of dark matter in the form of dark deuterons as a function of the red shift. Our results are summarized and discussed in section 6 .

\section{Universal two-body physics with large scattering length}

In this section, we summarize the universal two-body physics of particles with short-range self-interactions and a large scattering length. We determine the mass and the large scattering length of a dark nucleon that would be required to solve the small-scale structure problems of the universe.

\subsection{Two-body physics}

Atomic physics has provided a strong impetus for developing the universal few-body physics of particles with large scattering lengths [38]. There are naturally occurring atoms with large scattering lengths, such as the ${ }^{4} \mathrm{He}$ atom. There are other atoms whose scattering lengths can be controlled and made arbitrarily large by using Feshbach resonances [48]. In this subsection, we use the concise language of atomic physics for the particles with large scattering lengths and their bound clusters. The particle $d$ is referred to as an atom, and the two-body bound cluster $d_{2}$ is called a dimer. We make factors of Planck's constant $\hbar$ explicit.

We denote the mass of the atom $d$ by $m$. The atom has short-range self-interactions with range $r_{0}$ and an S-wave scattering length $a$ that is much larger than $r_{0}$. The range and the scattering length provide a high energy scale $E_{0}=\hbar^{2} / m r_{0}^{2}$ and a low energy scale $E_{2}=\hbar^{2} / m a^{2}$. At energies well below $E_{0}$, the two-body physics is universal in the sense that it is completely determined by $a$. It depends on the nature of the particles and on the details of their short-range interactions only through $a$. The universal behavior becomes exact in the zero-range limit $r_{0} \rightarrow 0$. In this limit, all higher partial-wave interactions go to 0 , so two-body scattering is purely S-wave.

The universal region for the scattering of two atoms is when the collision energy $E$, which is the kinetic energy in the center-of-mass frame, is well below $E_{0}$. The universal elastic scattering cross section for identical bosons is

$$
\sigma_{\text {elastic }}(E)=\frac{8 \pi}{1 / a^{2}+m E / \hbar^{2}} .
$$

If the two colliding atoms are distinguishable particles, such as the two spin states of a spin- $\frac{1}{2}$ fermion, the numerator is replaced by $4 \pi$. The cross section has dramatic energy dependence. When the collision energy $E$ decreases below $E_{0}$, the elastic cross section 
increases in accordance with eq. (2.1). In the scaling region $E_{2} \ll E \ll E_{0}$, the cross section nearly saturates the S-wave unitarity bound $8 \pi \hbar^{2} / m E$. As the energy decreases below $E_{2}$, the cross section levels off and approaches its maximum value $8 \pi a^{2}$ as $E \rightarrow 0$. In the limit $a \rightarrow \pm \infty$, the scaling behavior $8 \pi \hbar^{2} / m E$ extends down to arbitrarily low energy. Since this cross section saturates the S-wave unitarity bound, the limit $a \rightarrow \pm \infty$ is called the unitary limit.

A universal bound state is one that has properties determined by $a$. Its binding energy per pair of particles must be less than $E_{0}$. Whether or not there is a universal dimer $d_{2}$ depends on the sign of $a$. If $a<0$, there is no universal dimer. If $a>0$, there is a single universal dimer. The universal binding energy of $d_{2}$ in the zero-range limit is

$$
E_{2}=\hbar^{2} / m a^{2}
$$

A beautiful example in atomic physics of a boson with a large scattering length is the ${ }^{4} \mathrm{He}$ atom. Its scattering length is about $200 a_{0}$, where $a_{0}$ is the Bohr radius. The scattering length is larger than the effective range by about a factor of 15 , so the cross section increases at low energies by more than two orders of magnitude. The ${ }^{4} \mathrm{He}$ dimer is a universal two-body bound state with the tiny binding energy $E_{2}=1.4 \times 10^{-7} \mathrm{eV}$. The ${ }^{4} \mathrm{He}$ dimer was first observed in 1993 using electron impact ionization [49]. The universal low-energy behavior of particles with a large scattering length is illustrated even more dramatically by experiments with ultracold trapped atoms. The scattering length $a$ of the atoms can be controlled and made arbitrarily large by tuning the magnetic field to a Feshbach resonance [48]. Thus the binding energy of the universal dimer can be controlled and made arbitrarily small.

If the atoms have inelastic scattering channels, the scattering length $a$ is complex with a negative imaginary part. If all the inelastic scattering channels have energy release large compared to $E_{2}$, the inclusive inelastic cross section is also universal and determined by $a$. The universal inelastic scattering cross section for identical bosons is

$$
\sigma_{\text {inelastic }}(E)=\frac{8 \pi \operatorname{Im}[1 / a]}{\left(m E / \hbar^{2}\right)^{1 / 2}\left[1 / a^{2}+m E / \hbar^{2}\right]} .
$$

We have assumed the imaginary part of $1 / a$ is tiny compared to the real part of $1 / a$, in which case the imaginary part can be ignored except in the numerator where it appears as a multiplicative factor. Inelastic atom-atom scattering channels are also decay channels for the dimer. The universal expression for the decay rate is

$$
\Gamma_{2}=\frac{4 \hbar \operatorname{Im}[1 / a]}{m a}
$$

The imaginary part of $a$ should be ignored in the denominator. The energy $\hbar \Gamma_{2}$ is twice the imaginary part of the complex binding energy given by eq. (2.2) with complex $a$. Note that the imaginary part of $1 / a$ cancels in the ratio of the inelastic cross section in eq. (2.3) and the decay rate in eq. (2.4). 


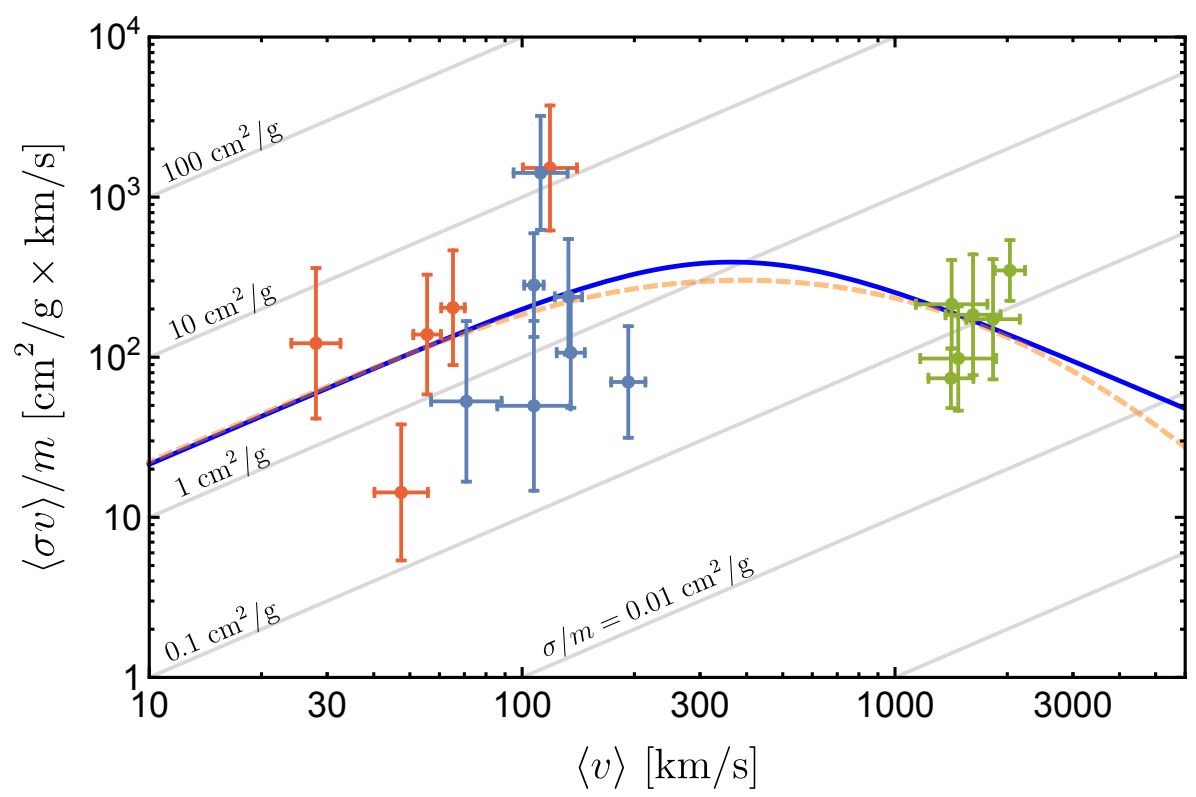

Figure 1. Self-interaction reaction rate $\left\langle v \sigma_{\text {elastic }}\right\rangle$ for dark matter particles as a function of the mean velocity $\langle v\rangle$. The data points are results from Kaplinghat, Tulin, and Yu for dwarf galaxies (red), low-surface-brightness galaxies (blue), and galaxy clusters (green) [53]. The curves are the best fit for a dark-photon model with $\alpha^{\prime}=1 / 137$ [53] (dashed) and the best fit to eq. (2.5) (solid). The diagonal lines are for energy-independent cross sections.

\subsection{Dark matter parameters}

The small-scale structure problems of the universe can be solved by self-interacting dark matter that becomes strongly interacting at low energies [46, 50-52]. In ref. [53], Kaplinghat, Tulin, and $\mathrm{Yu}$ determined self-interaction reaction rates $\left\langle v \sigma_{\text {elastic }}\right\rangle$ for dark matter particles from astrophysical data on dwarf galaxies, low-surface-brightness galaxies, and galaxy clusters [54-57]. Their data points are shown as a function of the mean relative velocity $\langle v\rangle$ of the dark atoms in figure 1. In the galaxies, $\langle v\rangle$ ranges from about $20 \mathrm{~km} / \mathrm{s}$ to about $200 \mathrm{~km} / \mathrm{s}$. The values of $\left\langle v \sigma_{\text {elastic }}\right\rangle$ for the galaxies only are roughly compatible with an energy-independent cross section with $\sigma_{\text {elastic }} / m=2 \mathrm{~cm}^{2} / \mathrm{g}$. In the galaxy clusters, $\langle v\rangle$ is about $2000 \mathrm{~km} / \mathrm{s}$. The values of $\left\langle v \sigma_{\text {elastic }}\right\rangle$ for the clusters only are compatible with an energy-independent cross section with $\sigma_{\text {elastic }} / m=0.1 \mathrm{~cm}^{2} / \mathrm{g}$. To fit the results for both the galaxies and the clusters requires a cross section that increases dramatically with decreasing velocity. The results for $\left\langle v \sigma_{\text {elastic }}\right\rangle$ versus $\langle v\rangle$ can be fit by a dark-photon model with three parameters: the dark matter mass $m_{\chi}$, the dark photon mass $\mu$, and the coupling constant $\alpha^{\prime}$ for a Yukawa potential. Kaplinghat et al. included additional systematic errors of 0.3 in $\log \left(\left\langle v \sigma_{\text {elastic }}\right\rangle / m\right)$ and 0.1 in $\log (\langle v\rangle)$ for each system to take into account the uncertainty in their modeling. They fixed the coupling constant at $\alpha^{\prime}=1 / 137$ and fit the parameters $m_{\chi}$ and $\mu$. Their fitted values are $m_{\chi}=15_{-5}^{+7} \mathrm{GeV}$ and $\mu=17 \pm 4 \mathrm{MeV}$. The curve for their best fit with $m_{\chi}=15 \mathrm{GeV}$ and $\mu=17 \mathrm{MeV}$ is shown in figure 1 . 
The results for the self-interaction reaction rates in ref. [53] can be fit equally well by a short-range interaction model with a large scattering length. We assume dark nucleons are identical spin- 0 bosons with a large real and positive scattering length. The parameters required to describe the universal two-body physics of dark nucleons are their mass $m_{\chi}$ and the scattering length $a$. The elastic cross section is given in eq. (2.1). The reaction rate as a function of the relative velocity $v$ is

$$
v \sigma_{\text {elastic }}(v)=\frac{8 \pi a^{2} v}{1+\left(a m_{\chi} / 2\right)^{2} v^{2}} .
$$

Our fit to the data points for $\left\langle v \sigma_{\text {elastic }}\right\rangle$ versus $\langle v\rangle$ shown in figure 1 gives

$$
\begin{aligned}
m_{\chi} & =19_{-2}^{+3} \mathrm{GeV}, \\
a & = \pm(17 \pm 3) \mathrm{fm} .
\end{aligned}
$$

The curve for the best fit with $m_{\chi}=19 \mathrm{GeV}$ and $a= \pm 17 \mathrm{fm}$ is shown in figure 1 . The binding energy $E_{2}=1 /\left(m_{\chi} a^{2}\right)$ of the dark deuteron is predicted to be $7.1 \mathrm{keV}$. This is also the value of the collision energy $m_{\chi} v^{2} / 4$ where $v \sigma_{\text {elastic }}(v)$ has a maximum as a function of $v$. The relative velocity $v$ at the maximum is about $300 \mathrm{~km} / \mathrm{s}$. The reaction rate in eq. (2.5) must remain accurate for $v$ beyond the values of $\langle v\rangle$ for galaxy clusters. At some larger velocity scale $v_{0}=2 / m_{\chi} r_{0}$ set by the range $r_{0}$ of self-interactions, the reaction rate in eq. (2.5) may cross over to that for an energy-independent cross section, which is a diagonal line in figure 1. Assuming the crossover does not occur until $v$ is at least 3 times larger than $\langle v\rangle$ for galaxy clusters, the energy-independent cross section must satisfy $\sigma_{\text {elastic }} / m_{\chi}<0.01 \mathrm{~cm}^{2} / \mathrm{g}$. The range $r_{0}$ must be less than $0.5 \mathrm{fm}$, and the energy scale $E_{0}=1 / m_{\chi} r_{0}^{2}$ set by the range must be greater than $200 \mathrm{MeV}$.

One can obtain a very similar curve in figure 1 with spin- 1 fermions, for which the factor $8 \pi$ in eq. (2.5) is replaced by $4 \pi$. The best-fit parameters for the mass and scattering length are $15 \mathrm{GeV}$ and $\pm 22 \mathrm{fm}$. This mass is the same as that obtained in the dark photon model of ref. [53]. The same scattering length could be obtained in that model by tuning either the dark photon mass or the Yukawa coupling constant. The mapping from the parameters of a model of dark fermions with gauge bosons to the scattering length is extensively discussed in refs. [58-60]. The mapping from the parameters of a more fundamental dark matter model with bosons to the scattering length could be as nontrivial as the mapping from the parameters of QCD to the large neutron scattering length.

An upper bound on the elastic cross section for dark matter particles has been obtained from the Bullet Cluster, which is the result of a collision of two galaxies with a relative velocity estimated to be $\mathcal{O}(1000) \mathrm{km} / \mathrm{s}$ [61-67]. The apparent absence of significant scattering from the two dark matter halos implies an upper bound on the elastic cross section for the dark matter particles divided by their mass. If the dark matter particles have an energyindependent cross section, the upper bound on $\sigma_{\text {elastic }} / m_{\chi}$ is roughly $1 \mathrm{~cm}^{2} / \mathrm{g}$ [68-70]. The curves in figure 1 at $\langle v\rangle=1000 \mathrm{~km} / \mathrm{s}$ are compatible with this bound.

Another constraint on the elastic cross section for dark matter particles can be obtained by demanding that self-scattering removes the cusp in the dark matter distribution 
at the center of dwarf galaxies that is predicted by the $\Lambda \mathrm{CDM}$ model. If the dark matter particles have an energy-independent cross section, this condition provides an estimate of $\sigma_{\text {elastic }} / m_{\chi}$ that is roughly $1 \mathrm{~cm}^{2} / \mathrm{g}$ [46]. A typical mean velocity of dark matter particles in a dwarf galaxy is $10 \mathrm{~km} / \mathrm{s}$. The curves in figure 1 at $\langle v\rangle=10 \mathrm{~km} / \mathrm{s}$ are compatible with this estimate.

Note that in our fit to eq. (2.5) we have only considered the data compiled by Kaplinghat et al. in ref. [53]. We are aware that there are analyses of astrophysical systems that exhibit agreement with collisionless dark matter (see e.g. refs. [68, 71]). There are caveats to these analyses, since the galaxy-dark matter offsets are predicted by strongly interacting dark matter are small [72] and since the concentration parameter of dwarf galaxies may be higher than assumed. ${ }^{1}$ Whether or not self-interacting dark matter is required in astrophysical systems requires more research. We will use the fit parameters in eq. (2.6) to illustrate the near-threshold S-wave resonance model, but we will also consider values of the parameters that do not solve the small-scale structure problems.

\section{Universal three-body physics of identical bosons}

In this section, we summarize the universal three-body physics of identical bosons with a large scattering length, which is surprisingly intricate [38]. The 3-body physics depends strongly on the scattering length $a$. It also depends log-periodically on a 3-body parameter $\kappa_{*}$ that can be determined from the binding energy of a universal bound 3-body cluster.

In this section, we use the concise language of atomic physics for the particles and their bound clusters. The particle $d$ is referred to as an atom. A two-body bound cluster $d_{2}$, a three-body bound cluster $d_{3}$, and a four-body bound cluster $d_{4}$ are called a dimer, a trimer, and a tetramer, respectively. We make factors of Planck's constant $\hbar$ explicit.

\subsection{Trimer spectrum}

The remarkable nature of trimers composed of identical bosons with a large scattering length was first realized by Vitaly Efimov. In 1970, Efimov pointed out that in the unitary limit where $a$ is infinitely large, there is a sequence of infinitely many trimers whose binding energies have an accumulation point at the 3-atom scattering threshold [73]. The ratio of the binding energies of two successive trimers is the square of a universal number $\lambda_{0}=22.694$. The order of magnitude of the binding energy of the most deeply bound Efimov trimer is the energy scale $E_{0}=\hbar^{2} / m r_{0}^{2}$ set by the range.

The discrete spectrum of Efimov trimers in the unitary limit $a= \pm \infty$ implies that few-body physics in the zero-range limit must depend on a 3-body parameter. The Efimov trimers can be labeled by an integer $n$. A convenient choice for the 3-body parameter is the binding wave number $\kappa_{*}$ in the unitary limit $a= \pm \infty$ of some arbitrarily chosen Efimov trimer labelled by $n_{*}$. In the unitary limit, the binding energies of the other Efimov trimers differ by integer powers of $\lambda_{0}^{2} \approx 515$ :

$$
E_{3, n}=\lambda_{0}^{-2\left(n-n_{*}\right)} \hbar^{2} \kappa_{*}^{2} / m \quad \text { at } \quad a= \pm \infty .
$$

\footnotetext{
${ }^{1}$ Private communication from M. Kaplinghat.
} 
If the binding wave number of a different Efimov trimer was chosen as the 3-body parameter, the value of $\kappa_{*}$ would differ by a multiplicative factor that is an integer power of $\lambda_{0}$. Since $\kappa_{*}$ can only be defined modulo multiplicative factors of $\lambda_{0}$, few-body physics can only depend log-periodically on $\kappa_{*}$. In particular, 3-body reaction rates must be functions of $a$ and $\kappa_{*}$ that are invariant under replacing $\kappa_{*}$ by $\lambda_{0} \kappa_{*}$.

The binding energies of Efimov trimers are smooth functions of the inverse scattering length $1 / a$ [74]. If the scattering length is not infinitely large, there are only a finite number of Efimov trimers. As $1 / a$ decreases through negative values, Efimov trimers disappear through the 3-atom scattering threshold at critical values of $a$ that differ by multiplicative factors of $\lambda_{0}$. As $1 / a$ increases through positive values, Efimov trimers disappear through the atom-dimer scattering threshold at critical values of $a$ that differ by multiplicative factors of $\lambda_{0}$. The Efimov trimer whose binding momentum in the unitary limit is $\kappa_{*}$ disappears through the 3-atom scattering threshold $E=0$ at the negative scattering length $a_{-}=-1.508 \kappa_{*}^{-1}$ [75], and it disappears through the atom-dimer scattering threshold $E=-E_{2}$ at the positive scattering length $a_{*} \approx 0.07076 \kappa_{*}^{-1}$ [38]. Given any large scattering length $a$, the energy of one Efimov trimer can be used to determine $\kappa_{*}$ and the binding energies of the other Efimov trimers can then be predicted. The number of Efimov trimers is not predicted, because the binding energy of the deepest Efimov trimer is determined by the range $r_{0}$.

In atomic physics, the two ${ }^{4} \mathrm{He}$ trimers are beautiful examples of Efimov trimers. The binding energy of the more deeply bound ${ }^{4} \mathrm{He}$ trimer is about $1.1 \times 10^{-5} \mathrm{eV}$. It was first observed using diffraction from a transmission grating [76]. The binding energy of the more weakly bound ${ }^{4} \mathrm{He}$ trimer relative to the 3 -atom threshold is about $2.3 \times 10^{-7} \mathrm{eV}$, which is about a factor of 2 larger than that of the ${ }^{4} \mathrm{He}$ dimer. It has been observed only recently using Coulomb explosion imaging [77]. The first Efimov trimer observed in cold atom physics was a ${ }^{133} \mathrm{Cs}$ trimer observed in 2008 as a resonance in the atom loss rate from 3-body recombination [78].

\subsection{Dimer-atom scattering}

The dimer-atom scattering processes are elastic scattering $\left(d_{2}+d \rightarrow d_{2}+d\right)$ and dimerbreakup scattering $\left(d_{2}+d \rightarrow d+d+d\right)$. The collision energy, which is the total kinetic energy of the atom and dimer in the center-of-momentum frame, is

$$
E=\frac{3 \hbar^{2} k^{2}}{4 m}
$$

where $\hbar k$ is the relative momentum of the atom and dimer. The partial wave expansion for the elastic scattering amplitude is

$$
f_{k}(\theta)=\sum_{J=0}^{\infty} \frac{2 J+1}{k \cot \delta_{J}(k)-i k} P_{J}(\cos \theta) .
$$

The phase shifts $\delta_{J}(k)$ are dimensionless functions of $k$. The scattering is purely elastic for energies between the atom-dimer threshold $E=0$ and the dimer-breakup threshold $E=E_{2}$. The phase shifts are therefore real for $E<E_{2}$ and complex for $E>E_{2}$. The 
cross sections for elastic scattering and for breakup scattering can be expressed in terms of the phase shifts:

$$
\begin{aligned}
\sigma_{\text {elastic }}(E) & =\frac{4 \pi}{k^{2}} \sum_{J=0}^{\infty}(2 J+1)\left|e^{i \delta_{J}(k)} \sin \delta_{J}(k)\right|^{2}, \\
\sigma_{\text {breakup }}(E) & =\frac{\pi}{k^{2}} \sum_{J=0}^{\infty}(2 J+1)\left(1-\left|e^{2 i \delta_{J}(k)}\right|^{2}\right) .
\end{aligned}
$$

In the universal regime where the energy $E$ is much smaller than the energy scale $E_{0}$ set by the range, the only relevant interaction parameters are the scattering length $a$ and the 3-body parameter $\kappa_{*}$. The S-wave phase shift $\delta_{0}(k)$ is a dimensionless function of $k a$ and $a \kappa_{*}$ that depends only log-periodically on $a \kappa_{*}$. It can be expressed in the form [38]

$$
\exp \left(2 i \delta_{0}(E)\right)=s_{22}(x)+\frac{s_{12}(x)^{2} \exp \left[2 i s_{0} \log \left(a / a_{+}\right)\right]}{1-s_{11}(x) \exp \left[2 i s_{0} \log \left(a / a_{+}\right)\right]},
$$

where $s_{0}=\pi / \log \lambda_{0} \approx 1.00624$ is a universal constant and $a_{+}$is an alternative 3-body parameter that differs from $\kappa_{*}^{-1}$ by a multiplicative factor: $a_{+}=0.3165 \kappa_{*}^{-1}$ [75]. The dimensionless functions $s_{11}(x), s_{12}(x)$, and $s_{22}(x)$ are complex-valued functions of the scaling variable $x=k a$. In terms of this variable, the dimer-breakup threshold $E=E_{2}$ is $x=2 / \sqrt{3}$. For $x<2 / \sqrt{3}$, the scaling functions $s_{11}(x), s_{12}(x)$, and $s_{22}(x)$ are entries of a $2 \times 2$ unitary matrix. For $x>2 / \sqrt{3}$, they are entries of a $2 \times 2$ submatrix of a $3 \times 3$ unitary matrix. In ref. [79], they were calculated numerically over the range of $x$ from $10^{-1}$ to $10^{+1}$. The phase shifts $\delta_{J}(k)$ for the higher partial waves are also dimensionless functions of $x=k a$. They are real for $x<2 / \sqrt{3}$ and complex for $x>2 / \sqrt{3}$. In ref. [79], the phase shifts for $J$ from 1 to 6 were calculated numerically over the range of $x$ from $10^{-1}$ to $10^{+1}$. The results of ref. [79] allow the elastic cross section and the breakup cross section to be calculated for collision energies up to $100 E_{2}$, where $E_{2}$ is the dimer binding energy in eq. (2.2).

The breakup cross section is shown in figure 2 as bands whose envelope corresponds to minimizing and maximizing the cross section with respect to $a_{+}$. The upper band is the total cross section, and the lower band is the contribution from $J=0$. The curves inside the lower band are for 8 values of $a_{+} / a$ between 1 and $\lambda_{0}$ that are equally spaced on a log scale. At $E=100 E_{2}$, the sum of the higher partial waves is larger than the maximum $J=0$ contribution by more than an order of magnitude. The behavior of the individual partial waves at large $E$ is consistent with decreasing as $1 / E^{2}$, but the sum of the partial waves is consistent with decreasing as $1 / E$. This is the scaling behavior of the cross section at high energy that is expected from dimensional analysis, given that the only energy scale from interactions is $E_{2}$. Both the upper and lower bands are extrapolated beyond $100 E_{2}$ by fitting to power-law scalings between $50 E_{2}$ and $100 E_{2}$. The breakup cross section in the high-energy limit can be approximated as

$$
\sigma_{\text {breakup }}(E) \rightarrow c_{2} \frac{\hbar^{2}}{m E},
$$

where the coefficient is $c_{2} \approx 35$. 


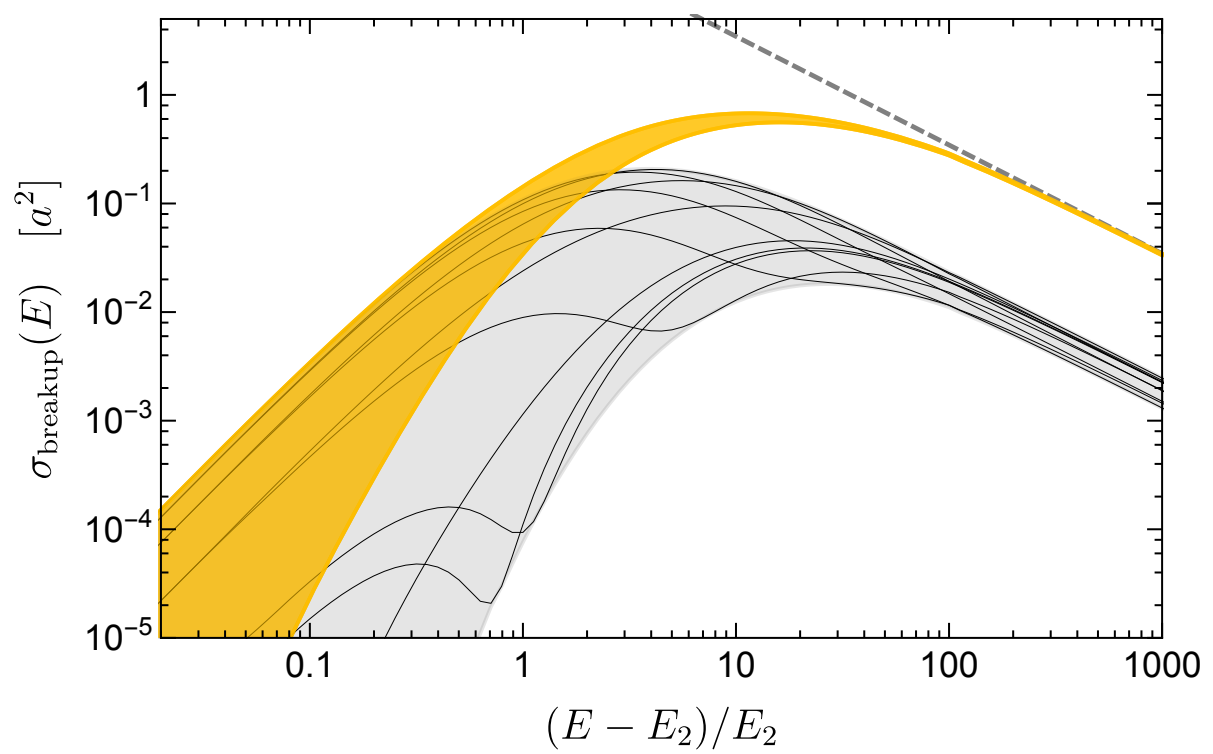

Figure 2. The breakup cross section as a function of energy with respect to the threshold: $E-E_{2}$. The upper band is the envelope of $\sigma_{\text {breakup }}(E)$ for all possible values of the three-body parameter $a_{+}$. The lower band is the envelope of the $J=0$ contribution to $\sigma_{\text {breakup }}(E)$ for all possible values of $a_{+}$. The curves inside the lower band are for 8 values of $a_{+}: a_{+} / a=\lambda_{0}^{n / 8}, n=0,1, \ldots, 7$. The dashed line is the extrapolation from the scaling behavior in eq. (3.6).

Analytic expressions for the cross sections are known at special values of the energy [38]. They can be useful for making order-of-magnitude estimates. The elastic cross section at the atom-dimer threshold is

$$
\sigma_{\text {elastic }}(E=0)=4 \pi\left(1.46+2.15 \cot \left[s_{0} \log \left(a / a_{*}\right)\right]\right)^{2} a^{2},
$$

where $a_{*}=0.0708 \kappa_{*}^{-1}$. The cross section at $E=0$ depends log-periodically on $a / a_{*}$, and its value ranges from 0 to $\infty$. It diverges at $a_{*}=a$ and at other values of $a_{*}$ that differ from $a$ by an integer power of $\lambda_{0}=22.7$, because there is an Efimov trimer at the atom-dimer threshold. The cross section at $E=0$ vanishes at $a_{*}=2.63 a$ and at other values of $a_{*}$ that differ from $2.63 a$ by an integer power of $\lambda_{0}$, because there is destructive interference between two scattering pathways. The S-wave contribution to the elastic cross section at the dimer-breakup threshold is known analytically. It can be approximated with an accuracy of better than $1 \%$ by [38]

$$
\sigma_{\text {elastic }}^{(J=0)}\left(E=E_{2}\right) \approx 3 \pi \sin ^{2}\left[s_{0} \log \left(a / a_{+}\right)\right] a^{2},
$$

where $a_{+} \approx 0.3165 \kappa_{*}^{-1}$. This contribution vanishes at $a_{+}=a$ and at other values of $a_{+}$ that differ from $a$ by an integer power of $\lambda_{0}$, because there is perfect destructive interference between two reaction pathways. The S-wave contribution to the breakup cross section at an energy $E$ just above the dimer-breakup threshold $E_{2}$ is [79]

$$
\sigma_{\text {breakup }}^{(J=0)}(E) \approx \frac{C_{3}\left(a / a_{+}\right)}{32 \sqrt{3} \pi}\left(\frac{E-E_{2}}{E_{2}}\right)^{2} a^{2} .
$$


The coefficient $C_{3}\left(a / a_{+}\right)$in the prefactor depends log-periodically on $a$. A completely analytic expression for this coefficient has been derived by Macek, Ovchinnikov, and Gasaneo [80]. It can be expressed as

$$
C_{3}\left(a / a_{+}\right)=\frac{66.6373 \sin ^{2}\left[s_{0} \log \left(a / a_{+}\right)\right]}{1-0.00717 \sin ^{2}\left[s_{0} \log \left(a / a_{+}\right)\right]},
$$

where $s_{0}=\pi / \log \lambda_{0}=1.00624$ and $a_{+} \approx 0.3165 \kappa_{*}^{-1}$ [75]. It vanishes at $a_{+}=a$ and at other values of $a_{+}$that differ from $a$ by an integer power of $\lambda_{0}=22.7$. At these values of $a_{+}$, there is perfect destructive interference between two recombination pathways. The coefficient in (3.10) can be approximated with an error of less than $1 \%$ by the simpler expression

$$
C_{3}\left(a / a_{+}\right) \approx 67.1 \sin ^{2}\left[s_{0} \log \left(a / a_{+}\right)\right]
$$

\subsection{Three-body recombination}

Three-body recombination is a reaction in which the collision of three atoms results in the formation of a dimer: $d+d+d \rightarrow d_{2}+d$. The reaction rate depends on the wave vectors $\boldsymbol{k}_{1}, \boldsymbol{k}_{2}$, and $\boldsymbol{k}_{3}$ of the three colliding atoms, but not on the total wave vector $\boldsymbol{k}_{1}+\boldsymbol{k}_{2}+\boldsymbol{k}_{3}$. It can be expressed as a function of the Jacobi wave vectors defined by $\boldsymbol{k}_{12}=\boldsymbol{k}_{1}-\boldsymbol{k}_{2}$ and $\boldsymbol{k}_{3,12}=\boldsymbol{k}_{3}-\frac{1}{2}\left(\boldsymbol{k}_{1}+\boldsymbol{k}_{2}\right)$. The collision energy $E$ is the kinetic energy in the center-of-momentum frame:

$$
E=\frac{\hbar^{2}\left(3 k_{12}^{2}+4 k_{3,12}^{2}\right)}{12 m}
$$

The recombination rate can be expressed as a function of $E$ and 5 dimensionless hyperangles consisting of the spherical angles of the two Jacobi vectors and $\arctan \left(\sqrt{3} k_{12} / 2 k_{3,12}\right)$. The hyperangular average of the recombination rate is a function of $E$ only. It can be expressed in terms of the breakup cross section at the kinetic energy $E_{2}+E$ [79]:

$$
\left\langle R\left(\boldsymbol{k}_{12}, \boldsymbol{k}_{3,12}\right)\right\rangle=\frac{192 \sqrt{3} \pi \hbar^{3}\left(E_{2}+E\right)}{m^{2} E^{2}} \sigma_{\text {breakup }}\left(E_{2}+E\right) .
$$

In the universal regime where the collision energy is much smaller than the energy scale $\hbar^{2} / m r_{0}^{2}$ set by the range, the only relevant interaction parameters are the scattering length $a$ and the 3-body parameter $\kappa_{*}$. A completely analytic expression for the three-body recombination rate at zero collision energy has been derived by Macek, Ovchinnikov, and Gasaneo [80]. It can be expressed as

$$
R(E=0)=6 C_{3}\left(a / a_{+}\right) \hbar a^{4} / m .
$$

The coefficient $C_{3}\left(a / a_{+}\right)$depends log-periodically on $a / a_{+}$, and it can be approximated by the expression in eq. (3.11). The recombination rate at $E=0$ vanishes at $a_{+}=a$ and at other values of $a_{+}$that differ from $a$ by an integer power of $\lambda_{0}$. It has its maximum value $67.1 \hbar a^{4} / m$ at $a_{+}=4.76 a$ and at other values of $a_{+}$that differ from $4.76 a$ by an integer power of $\lambda_{0}$. 


\subsection{Four-body physics and beyond}

In 2004, Platter, Hammer, and Meissner predicted the existence of universal 4-body bound clusters composed of identical bosons with large scattering length [81, 82]. Their binding energies were mapped out as functions of $a$ by von Stecher, D'Incao, and Greene [83]. In an experiment with ${ }^{133} \mathrm{Cs}$ atoms in 2009 , the dramatic increase of the 4-body recombination rate at low temperature near a specific value of $a$ was used to discover the first such universal tetramer [84]. There is theoretical evidence for universal bound clusters of 5,6 , and even more identical bosons with a large scattering length [85].

\section{Rate coefficients at thermal equilibrium}

In this section, we give expressions for the rate coefficients for few-body reactions for identical bosons with large scattering lengths in thermal equilibrium. We use the concise language of atomic physics for the bosons and their bound clusters. We consider a gas of atoms with number density $n_{1}$ and dimers with number density $n_{2}$ in kinetic equilibrium at temperature $T$ but not necessarily in chemical equilibrium. For simplicity, we assume the gas is sufficiently dilute that the Bose-Einstein momentum distributions of the atoms and dimers can be approximated by Maxwell-Boltzmann distributions.

\subsection{Inelastic atom-atom scattering}

We assume all the inelastic atom-atom scattering channels have energy release large compared to $E_{2}$, and that the energetic particles produced by the reaction have scattering cross sections with an atom that are small compared to the elastic atom-atom cross section. The particles produced by an inelastic reaction can therefore be ignored, and the only effect of the reaction is to decrease the number of atoms by 2 . In a homogeneous system, the rate at which the number density $n_{1}$ of atoms decreases from inelastic atom-atom scattering is proportional to $n_{1}^{2}$ :

$$
\frac{d}{d t} n_{1}=-2 K_{1}(T) n_{1}^{2}
$$

The rate coefficient $K_{1}(T)$ depends on the temperature and can be expressed as a weighted integral over the inelastic cross section:

$$
K_{1}(T)=\frac{4}{\sqrt{\pi m}(k T)^{3 / 2}} \int_{0}^{\infty} d E E e^{-E / k T} \sigma_{\text {inelastic }}(E) .
$$

Upon inserting the universal approximation to the inelastic cross section for identical bosons in eq. (2.3), we obtain an analytic result:

$$
K_{1}(T)=\left(32 \pi g\left(k T / E_{2}\right) \frac{E_{2}}{k T}\right) \frac{\hbar \operatorname{Im}[a]}{m},
$$

where the dimensionless function $g(t)$ is $1-(\pi / t)^{1 / 2} e^{1 / t}[1-\operatorname{erf}(1 / \sqrt{t})]$. Figure 3 shows the rate coefficient $K_{1}(T)$ and its limiting behaviors: $16 \pi \hbar \operatorname{Im}[a] / m$ in the low- $T$ limit and $32 \pi\left(E_{2} / k T\right) \hbar \operatorname{Im}[a] / m$ in the high- $T$ limit. 


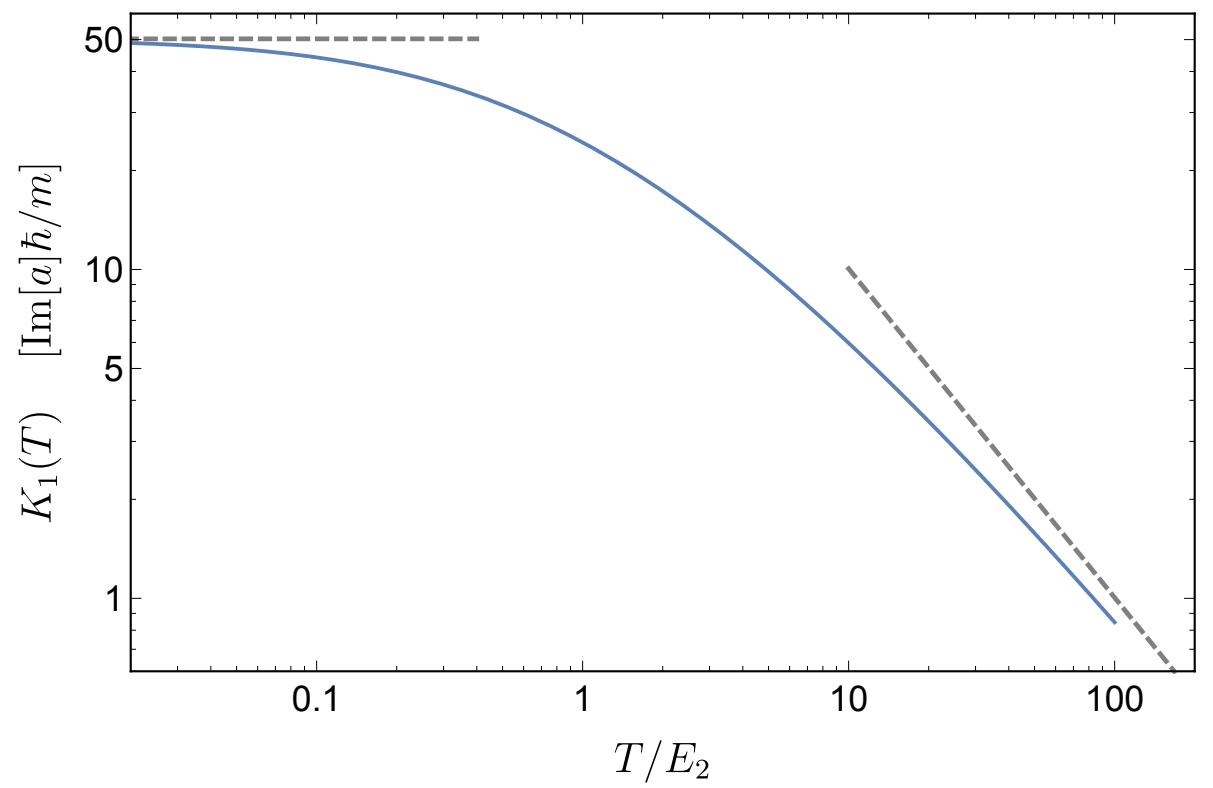

Figure 3. Rate coefficient $K_{1}(T)$ for dimer breakup as a function of the temperature $T$. The dashed lines indicate its asymptotic behaviors in the low- $T$ and high- $T$ limits.

\subsection{Dimer breakup}

The dimer-breakup reaction $d d_{2} \rightarrow d d d$ decreases the number of dimers by 1 and increases the number of atoms by 2 . We assume the final-state atoms are thermalized by the elastic atom-atom scattering. In a homogeneous system, the rate at which the number density $n_{2}$ of dimers decreases from dimer-breakup scattering is proportional to $n_{1} n_{2}$ :

$$
\frac{d}{d t} n_{2}=-K_{2}(T) n_{1} n_{2}
$$

The rate coefficient $K_{2}(T)$ depends on the temperature and can be expressed as a Boltzmann average of the dimer-breakup cross section:

$$
K_{2}(T)=\frac{6}{\sqrt{3 \pi m}(k T)^{3 / 2}} \int_{E_{2}}^{\infty} d E E e^{-E / k T} \sigma_{\text {breakup }}(E) .
$$

The universal approximation to the dimer-breakup cross section is given in eq. (3.4). The universal results for the atom-dimer phase shifts $\delta_{J}(k)$ in ref. [79] are obtained up to about $100 E_{2}$. The breakup cross section is extended above $100 E_{2}$ as shown in figure 2 by fitting to the power-law behavior in eq. (3.6). This allows $K_{2}(T)$ to be calculated for all temperatures $k T$ up to the scale $E_{0}$ set by the range. The results for the rate coefficient are shown in figure 4 as bands whose envelope corresponds to minimizing and maximizing the rate with respect to $a_{+}$. The upper band is the total rate coefficient, and the lower band is the contribution from $J=0$. The curves inside the lower band are for 8 values of $a_{+} / a$ between 1 and $\lambda_{0}$ that are equally spaced on a log scale.

We can obtain a simple analytic approximation for $K_{2}(T)$ in the low-temperature limit $k T \ll E_{2}$. In this limit, the breakup cross section in eq. (3.9) is dominated by the S-wave 


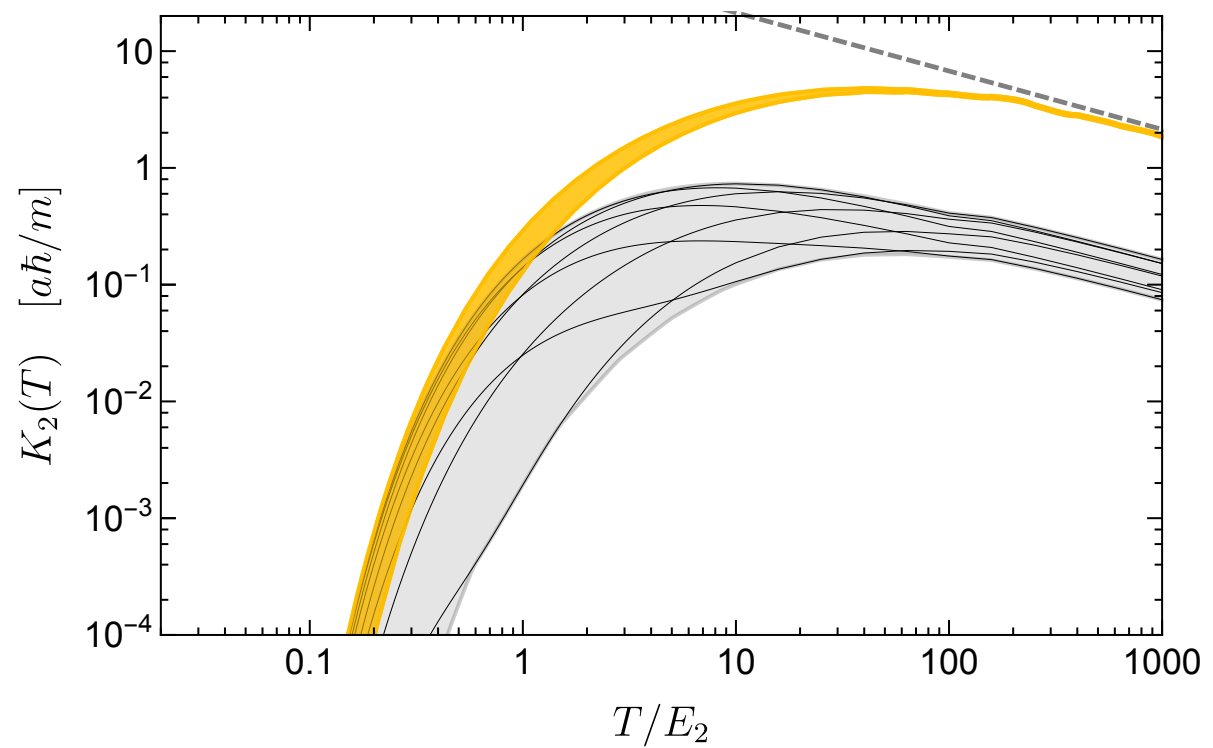

Figure 4. Rate coefficient $K_{2}(T)$ for dimer breakup as a function of the temperature $T$. The upper band is the envelope of $K_{2}(T)$ for all possible values of the three-body parameter $a_{+}$. The dashed line is the extrapolation from the scaling behavior in eq. (4.7). The lower band is the envelope of the $J=0$ contribution to $K_{2}(T)$ for all possible values of $a_{+}$. The curves inside the lower band are for 8 values of $a_{+}: a_{+} / a=\lambda_{0}^{n / 8}, n=0,1, \ldots, 7$.

contribution. The limiting behavior of the rate coefficient is

$$
K_{2}(T) \longrightarrow \frac{C_{3}\left(a / a_{+}\right)}{2 \sqrt{2}} e^{-E_{2} / k T}\left(\frac{a}{\lambda_{T}}\right)^{3} \frac{\hbar a}{m},
$$

where $\lambda_{T}=\left(2 \pi \hbar^{2} / m k T\right)^{1 / 2}$ is the thermal wavelength and $C_{3}\left(a / a_{+}\right)$is the coefficient of $\hbar a^{4} / m$ in the 3 -body recombination rate at zero collision energy in eq. (3.14). This coefficient can be accurately approximated by eq. (3.11). Note that the dimer-breakup rate coefficient in eq. (4.6) is exponentially suppressed by the Boltzmann factor.

We can also obtain a simple analytic approximation for $K_{2}(T)$ in the scaling region, where $k T$ is much larger than $E_{2}$ and much smaller than the energy scale $E_{0}=\hbar^{2} / m r_{0}^{2}$ set by the range. In the scaling region $E_{2} \ll k T \ll E_{0}$, the breakup cross section in eq. (3.9) is dominated by the higher partial-wave contributions. Figure 4 shows that at $k T=100 E_{2}$, the sum of the higher partial waves is already more than an order of magnitude larger than the maximum $J=0$ contribution. The dependence on the S-wave scattering length $a$ can therefore be neglected. Since the interactions provide no other length scales smaller than the range, the dependence of the rate coefficient on $T$ can be determined up to a numerical coefficient by dimensional analysis:

$$
K_{2}(T) \longrightarrow c_{2} \frac{\sqrt{6}}{\pi} \frac{\hbar \lambda_{T}}{m},
$$

where $c_{2} \approx 35$ is the same coefficient as in eq. (3.6). The extrapolation in $T$ provided by the scaling behavior in eq. (4.7) is shown as a dashed line in figure 4 . 


\subsection{Three-body recombination}

The three-body recombination reaction $d d d \rightarrow d d_{2}$ increases the number of dimers by 1 and decreases the number of atoms by 2 . We assume the final-state atom and the finalstate dimer are thermalized by elastic atom-atom scattering and by elastic atom-dimer scattering, respectively. In a homogeneous system, the rate at which the number density $n_{2}$ of dimers increases from 3 -body recombination is proportional to $n_{1}^{3}$ :

$$
\frac{d}{d t} n_{2}=+K_{3}(T) n_{1}^{3}
$$

The rate coefficient $K_{3}(T)$ depends on the temperature and can be expressed as a Boltzmann average of the three-body recombination rate:

$$
K_{3}(T)=\frac{\int_{0}^{\infty} d E E^{2} e^{-E / k T}\left\langle R\left(\boldsymbol{k}_{12}, \boldsymbol{k}_{3,12}\right)\right\rangle}{6 \int_{0}^{\infty} d E E^{2} e^{-E / k T}}
$$

where $\langle R\rangle$ is the hyperangular average of the 3-body recombination rate, which is a function of the collision energy $E$ only. The factor of $1 / 3$ ! compensates for the overcounting of 3body states of the 3 identical bosons in the Boltzmann average. The rate coefficient can be expressed as a weighted integral over the dimer-breakup cross section:

$$
K_{3}(T)=\frac{16 \sqrt{3} \pi \hbar^{3}}{m^{2}(k T)^{3}} \int_{0}^{\infty} d E e^{-E / k T}\left(E_{2}+E\right) \sigma_{\text {breakup }}\left(E_{2}+E\right) .
$$

We can use eq. (4.5) to express $K_{3}(T)$ in terms of $K_{2}(T)$ :

$$
K_{3}(T)=2 \sqrt{2} \lambda_{T}^{3} e^{E_{2} / k T} K_{2}(T),
$$

where $\lambda_{T}=\left(2 \pi \hbar^{2} / m k T\right)^{1 / 2}$ is the thermal wavelength. The universal approximation to the dimer-breakup cross section is given in eq. (3.4). The universal results for the atomdimer phase shifts $\delta_{J}(k)$ in ref. [79] are obtained up to about $100 E_{2}$ and the breakup cross section is extended above $100 E_{2}$ in figure 2 by fitting to the power-law behavior. This allows the recombination rate coefficient $K_{3}(T)$ to be calculated for all temperatures $k T$ up to scale $E_{0}$ set by the range. The results are shown in figure 5 as bands whose envelopes corresponding to minimizing and maximizing the rates with respect to $a_{+}$. The upper band is the total rate coefficient, and the lower band is the contribution from $J=0$. The curves inside the lower band are for 8 values of $a_{+} / a$ between 1 and $\lambda_{0}$ that are equally spaced on a $\log$ scale.

We can obtain simple analytic approximations for the 3-body recombination rate coefficient by using the relation between $K_{2}(T)$ and $K_{3}(T)$ in eq. (4.11) and the analytic approximations for $K_{2}(T)$ in eqs. (4.6) and (4.7). In the low-temperature limit $k T \ll E_{2}$, the rate coefficient approaches a constant that depends log-periodically on $a / a_{+}$:

$$
K_{3}(T) \longrightarrow C_{3}\left(a / a_{+}\right) \frac{\hbar a^{4}}{m}
$$




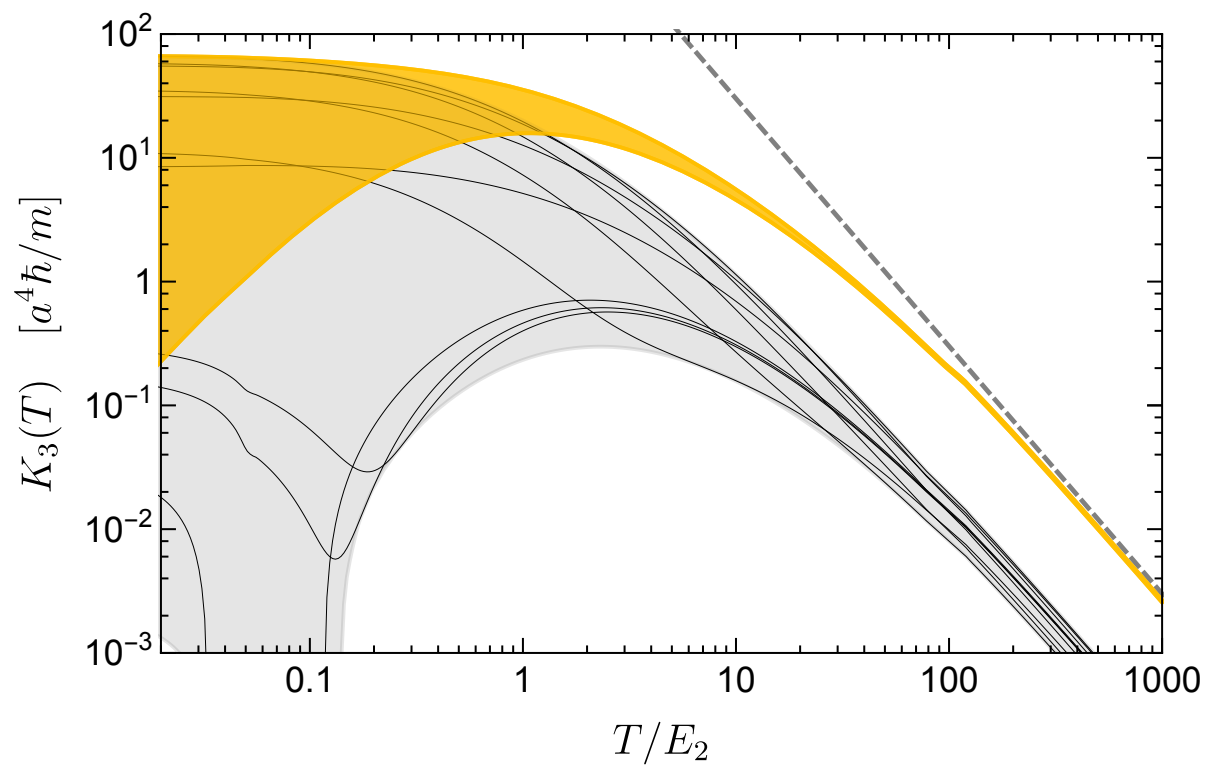

Figure 5. Rate coefficient $K_{3}(T)$ for three-body recombination as a function of the temperature $T$. The upper band is the envelope of $K_{3}(T)$ for all possible values of the three-body parameter $a_{+}$. The dashed line is the extrapolation from the scaling behavior in eq. (4.13). The lower band is the envelope of the $J=0$ contribution to $K_{3}(T)$ for all possible values of $a_{+}$. The curves inside the lower band are for 8 values of $a_{+}: a_{+} / a=\lambda_{0}^{n / 8}, n=0,1, \ldots, 7$.

where $C_{3}\left(a / a_{+}\right)$is the coefficient in eq. (3.10), which can be accurately approximated by eq. (3.11). In the scaling region $E_{2} \ll k T \ll E_{0}$, the rate coefficient scales as the power of temperature required by dimensional analysis:

$$
K_{3}(T) \longrightarrow c_{2} \frac{4 \sqrt{3}}{\pi} \frac{\hbar \lambda_{T}^{4}}{m},
$$

where $c_{2} \approx 35$ is the same coefficient as in eq. (3.6). The extrapolation in $T$ provided by the scaling behavior in eq. (4.13) is shown as a dashed line in figure 5 .

In experiments with ultracold trapped atoms, the atoms form an extremely dilute gas in the sense that the typical interatom spacing is much larger than the range of the interactions between atoms: $\left\langle n_{1}\right\rangle^{1 / 3} r_{0} \ll 1$, where $\left\langle n_{1}\right\rangle$ is the density-weighted average of the number density. Three-body recombination can be important in these experiments, because the dimer and atom in the final state often have enough kinetic energy to escape from the trapping potential. In that case, every recombination event results in the loss of three atoms. In an experiment with ${ }^{133} \mathrm{Cs}$ atoms in 2005, the dramatic increase of the 3-body recombination rate at low temperature when the scattering length was tuned to near the negative value $a_{-}=-1.5 \kappa_{*}^{-1}$ was used to discover an Efimov trimer [78].

\section{Early universe}

In this section, we study the production of dark deuterons through three-body recombination of dark nucleons during the Hubble expansion of the early universe under the assumption that the dark nucleons are identical bosons with a large positive scattering 
length. We calculate the fraction of dark matter in the form of dark deuterons as a function of the redshift.

\subsection{Rate equations}

After the decoupling of dark matter from ordinary matter, the densities of dark nucleons and larger dark nuclei evolve in thermal equilibrium until they are captured by the gravitational potential wells of galaxies. The time evolution is due to the Hubble expansion and to reactions among the dark nuclei. Assuming that the larger dark nuclei are weakly bound, the density and temperature at decoupling are large enough that any larger dark nucleus that is formed is immediately broken up by a collision with a dark nucleon. Thus we can take as an initial condition that the dark matter consists entirely of dark nucleons at the decoupling time.

Given an initial state consisting only of dark nucleons, larger dark nuclei can be formed by $N$-body recombination reactions in which $N$ dark nuclei collide and some of them form bound states. At sufficiently low dark nucleon number density $n_{1}$, the $N$-body recombination rate is proportional to $n_{1}^{N}$. Thus if a dark deuteron $d_{2}$ exists, the most favorable reaction is 3-body recombination $\left(d+d+d \leftrightarrow d_{2}+d\right)$. Once dark deuterons have been produced, larger dark nuclei can be formed by rearrangement collisions, such as $d_{n}+d_{2} \rightarrow d_{n+1}+d$. The formation of dark deuterons is a bottleneck that must be overcome by 3-body recombination in order to form the larger dark nuclei. We wish to determine whether this bottleneck can be overcome in the early universe when the dark matter is still in thermal equilibrium. To answer this, we can ignore dark nuclei $d_{n}$ with $n \geq 3$ and consider only the time evolution for dark nucleons and dark deuterons. The only reactions we need to take into account are 3-body recombination and the dark deuteron breakup reaction $\left(d_{2}+d \leftrightarrow d+d+d\right)$. We wish to determine whether a significant population of dark deuterons can be generated in the early universe.

We denote the number densities of the dark nucleon and the dark deuteron by $n_{1}(t)$ and $n_{2}(t)$. We assume the number densities of dark nuclei with larger dark baryon number are negligible, so the total dark baryon number density is

$$
n_{\text {dark }}(t)=n_{1}(t)+2 n_{2}(t) .
$$

The time evolution equations for $n_{1}(t)$ and $n_{2}(t)$ obtained from the Boltzmann equation are

$$
\begin{aligned}
& \left(\frac{d}{d t}+3 H\right) n_{1}=-2 K_{3}(T) n_{1}^{3}+2 K_{2}(T) n_{1} n_{2}-2 K_{1}(T) n_{1}^{2}, \\
& \left(\frac{d}{d t}+3 H\right) n_{2}=K_{3}(T) n_{1}^{3}-K_{2}(T) n_{1} n_{2}-\Gamma_{2} n_{2},
\end{aligned}
$$

where $H$ is the Hubble function, $K_{3}(T), K_{2}(T)$, and $K_{1}(T)$ are temperature-dependent event rate coefficients, and $\Gamma_{2}$ is the dark deuteron decay rate. The Hubble function $H(t)$ depends on time, being determined by the scale factor $a(t)$ of the universe: $H=d \ln (a) / d t$. The rate coefficients are functions of the temperature $T(t)$ of the dark matter, which also depends on time. 
We neglect the effects of the annihilation of dark nucleons into ordinary matter. We therefore set $K_{1}(T)=0$ and $\Gamma_{2}=0$ in the rate equations in eqs. (5.2). If there were such an annihilation process, it would decrease $n_{1}$ through annihilation collisions of two dark nucleons and it would decrease $n_{2}$ through the annihilation of the two constituents of the dark deuteron. The rates for both processes are determined by the same parameter $\operatorname{Im}[1 / a]$, which appears as a multiplicative parameter in both $K_{1}(T)$ and $\Gamma_{2}$. When $\Gamma_{2}$ is much larger than $3 H(t)$, the number density $n_{2}$ of dark deuterons decreases exponentially. Any dimers that have been produced by 3-body recombination would decay quickly on a cosmological time scale. The net effect is that $n_{2}$ would remain essentially 0 , and the decrease in $n_{1}$ would be given by the 3-body recombination term in eq. (5.2a) only. Since we ignore the annihilation of dark nucleons, our results for the number density of dark deuterons can be interpreted as upper bounds.

If we ignore the annihilation terms in the evolution equations in eqs. (5.2), we get a simpler equation for the total dark baryon number density:

$$
\left(\frac{d}{d t}+3 H(t)\right) n_{\text {dark }}(t)=0
$$

Using $d t=H^{-1} d \ln a$, the solution is

$$
n_{\text {dark }}(t)=n_{\text {dark }}(0)\left(\frac{a(0)}{a(t)}\right)^{3} .
$$

The time evolution of the total dark baryon number density does not depend on the dark matter interactions; it is just diluted by the Hubble expansion.

It is convenient to use the redshift $z$ as an alternative time variable. The redshift is related to the scale factor $a$ by $1+z(t)=a(0) / a(t)$. The solution for the total dark baryon number density in eq. (5.4) can be expressed as

$$
n_{\text {dark }}(z)=\frac{\rho_{\mathrm{cdm}}}{m_{\chi}}(1+z)^{3},
$$

where $\rho_{\mathrm{cdm}}=2.23 \times 10^{-30} \mathrm{~g} / \mathrm{cm}^{3}$ is the present average mass density of dark matter in the universe [86] and $m_{\chi}$ is the mass of the dark nucleon. The Hubble function in terms of redshift is given by

$$
H(z)=H_{0}\left[\Omega_{\gamma}(1+z)^{4}+\Omega_{m}(1+z)^{3}+\Omega_{\Lambda}\right]^{1 / 2},
$$

where the Hubble constant is $H_{0}=67.8 \mathrm{~km} \mathrm{~s}^{-1} \mathrm{Mpc}^{-1}$ and the fractions of the critical density of the Universe for CMB photons $\left(\Omega_{\gamma}\right)$, matter $\left(\Omega_{m}\right)$, and dark energy $\left(\Omega_{\Lambda}\right)$ are $5.38 \times 10^{-5}, 0.308$, and 0.692 , respectively [86].

Since the dark nucleons are nonrelativistic after the decoupling, their temperature $T(z)$ is proportional to the square of their average momentum [87]. On the other hand, the temperature $T_{\gamma}(z)$ of the photons is proportional to their average momentum. The Hubble expansion changes the momentum of a particle by a factor of $1+z$. Thus the two temperatures are different functions of the redshift:

$$
\begin{aligned}
T(z) & \approx T(0)(1+z)^{2}, \\
T_{\gamma}(z) & \approx T_{\mathrm{cmb}}(1+z),
\end{aligned}
$$


where $T(0)$ is the present temperature of dark matter that has not been captured by gravitational potential wells and $T_{\mathrm{cmb}}=2.73 \mathrm{~K}$ is the present temperature of the photons. At decoupling, the dark matter and ordinary matter are in thermal equilibrium: $T\left(z_{\mathrm{dc}}\right)=$ $T_{\gamma}\left(z_{\mathrm{dc}}\right)$, where $z_{\mathrm{dc}}$ is the redshift at decoupling. We are not displaying the dependence on the Standard Model degrees of freedom in these and the following expressions for the temperature for simplicity. The variation due to the three-body parameter is larger than the effects from the decreasing number of relativistic degrees of freedom. The dark matter temperature is therefore

$$
T(z) \approx T_{\mathrm{cmb}} \frac{(1+z)^{2}}{1+z_{\mathrm{dc}}} .
$$

The decoupling redshift can be expressed as $z_{\mathrm{dc}} \approx T\left(z_{\mathrm{dc}}\right) / T_{\mathrm{cmb}}$. If the thermal decoupling of dark matter and ordinary matter occurs not long after their chemical decoupling, the decoupling temperature is given approximately by the dark-matter mass multiplied by a constant: $k T\left(z_{\mathrm{dc}}\right) \approx m_{\chi} / 20$ [88]. The resulting estimate for the decoupling redshift is

$$
1+z_{\mathrm{dc}} \approx \frac{m_{\chi} / 20}{k T_{\mathrm{cmb}}} .
$$

The mass fraction of the dark matter in the form of dark deuterons is

$$
f_{2}(z)=2 n_{2}(z) / n_{\text {dark }}(z) .
$$

If we ignore the annihilation terms in the evolution equations in eqs. (5.2), the dark deuteron fraction satisfies the differential equation

$$
\frac{d}{d z} f_{2}=\frac{1}{(1+z) H}\left[-2 K_{3}(T) n_{\text {dark }}^{2}\left(1-f_{2}\right)^{3}+K_{2}(T) n_{\text {dark }} f_{2}\left(1-f_{2}\right)\right] .
$$

We have used $d t=-[(1+z) H]^{-1} d z$ to replace the time derivative by a redshift derivative. Given $H(z), n_{\text {dark }}(z)$, and $T(z)$ in eqs. (5.6), (5.5), and (5.8), our problem reduces to solving this single differential equation for $f_{2}(z)$ subject to the initial condition $f_{2}\left(z_{\mathrm{dc}}\right)=0$, where $z_{\text {dc }}$ is given in eq. (5.9).

\subsection{Approximation in scaling and threshold regions}

The evolution equation for the dark deuteron fraction with redshift in eq. (5.11) involves the rate coefficients $K_{2}(T)$ and $K_{3}(T)$. If dark nucleons are identical bosons with a large positive scattering length and if $k T$ is much smaller than the energy scale $E_{0}$ set by the range, the rate coefficients are given in eqs. (4.5) and (4.10). The rate coefficients have simple behavior in the low-temperature limit $k T \ll E_{2}$, where $E_{2}=1 /\left(m_{\chi} a^{2}\right)$ is the dark deuteron binding energy, and in the scaling region $E_{2} \ll k T \ll E_{0}$, where $E_{0}=1 /\left(m_{\chi} r_{0}^{2}\right)$ is the energy scale set by the range. We can use those results to determine the qualitative behavior of the dark deuteron fraction in those regions.

In the scaling region $E_{2} \ll k T \ll E_{0}$, the rate coefficients $K_{2}(T)$ and $K_{3}(T)$ have the limiting behaviors given in eqs. (4.7) and (4.13). They scale as $\lambda_{T}$ and $\lambda_{T}^{4}$, respectively, where $\lambda_{T}$ is the thermal wavelength, which is proportional to $(1+z)^{-1}$ :

$$
\lambda_{T}=\left(\frac{2 \pi\left(1+z_{\mathrm{dc}}\right)}{m_{\chi} k T_{\mathrm{cmb}}}\right)^{1 / 2} \frac{1}{1+z} .
$$


Since $n_{\text {dark }}$ is proportional to $(1+z)^{3}$, the products $K_{2} n_{\text {dark }}$ and $K_{3} n_{\text {dark }}^{2}$ are both proportional to $(1+z)^{2}$. Thus there can be an equilibrium value of $f_{2}$ for which the two terms on the right side of eq. (5.11) cancel. The ratio of $K_{3}(T)$ and $K_{2}(T)$ is given in eq. (4.11). The equilibrium fraction satisfies

$$
\frac{f_{2}}{\left(1-f_{2}\right)^{2}}=4 \sqrt{2}\left(\frac{2 \pi\left(1+z_{\mathrm{dc}}\right)}{m_{\chi} k T_{\mathrm{cmb}}}\right)^{3 / 2} \frac{\rho_{\mathrm{cdm}}}{m_{\chi}} .
$$

If the equilibrium value of $f_{2}$ is much less than 1 , it can be approximated by the right side of eq. (5.13). Upon inserting the estimate for the decoupling redshift in eq. (5.9), the right side reduces to $\left(\rho_{\mathrm{cdm}} / m_{\chi}\right) /\left(k T_{\mathrm{cmb}}\right)^{3}$ multiplied by a numerical constant. Since $\rho_{\mathrm{cdm}} /\left(k T_{\mathrm{cmb}}\right)^{3}=0.74 \mathrm{eV}$, the equilibrium value of $f_{2}$ is tiny as long as $m_{\chi}$ is orders of magnitude larger than $1 \mathrm{eV}$.

In the low-temperature region $k T \ll E_{2}$, the rate coefficients $K_{2}(T)$ and $K_{3}(T)$ have the limiting behaviors given in eqs. (4.6) and (4.12). They are proportional to $\lambda_{T}^{-6} e^{-\lambda_{T}^{2} / a^{2}}$ and $\lambda_{T}^{-6}$, respectively. The dark deuteron breakup is exponentially suppressed by the Boltzmann factor, so the breakup term in the rate equation can be dropped. If the value of $f_{2}$ is much less than 1 , we need to keep only the leading terms in $f_{2}$ in the recombination term in eq. (5.11). The rate equation then simplifies to

$$
\frac{d}{d z} f_{2}=-2 K_{3}(0) \frac{n_{\mathrm{dark}}^{2}}{(1+z) H} .
$$

When $z \gg 10^{4}$, the Hubble function in eq. (5.6) can be approximated as $H(z) \approx H_{0} \Omega_{\gamma}^{1 / 2} z^{2}$. The solution of eq. (5.14) is then

$$
f_{2}(z)=f_{2}(0)-C_{3}\left(a / a_{+}\right) \frac{a^{4} \rho_{\mathrm{cdm}}^{2}}{2 H_{0} \Omega_{\gamma}^{1 / 2} m_{\chi}^{3}} z^{4},
$$

where $f_{2}(0)$ is the present dark-deuteron fraction for dark matter that has not been captured by gravitational potential wells and $C_{3}\left(a / a_{+}\right)$can be approximated by eq. (3.11). The approach to $f_{2}(0)$ is predicted to be $z^{4}$ multiplied by a coefficient whose dependence on $a$ is $C_{3}\left(a / a_{+}\right) a^{4}$. The value of $f_{2}(0)$ should be determined by a boundary condition from the region of larger $z$ where $k T(z)$ is comparable to $E_{2}$. We are unable to determine $f_{2}(0)$ analytically, but it should depend log-periodically on the three-body parameter $a_{+}$. Our numerical results for $f(0)$ are consistent with an expression linear in $C_{3}$.

The evolution equation for the dark deuteron fraction $f_{2}(z)$ in eq. (5.11) with the rate coefficients $K_{2}(T)$ and $K_{3}(T)$ in eqs. (4.5) and (4.10) applies all the way back to the decoupling redshift provided the decoupling temperature is smaller than the scale $E_{0}$ set by the range: $k T\left(z_{\mathrm{dc}}\right)<E_{0}$. If $E_{0}$ is smaller than $k T\left(z_{\mathrm{dc}}\right)$, the simple expressions for $K_{2}(T)$ and $K_{3}(T)$ in eqs. (4.5) and (4.10) are not applicable until $k T$ decreases to below $E_{0}$. However once $k T$ enters the scaling region $E_{2} \ll k T \ll E_{0}, f_{2}$ will be driven quickly to the equilibrium value given by eq. (5.13). Thus the present value of $f_{2}$ is completely determined by the scattering length $a$ and the 3-body parameter $a_{+}$provided only that the decoupling temperature is much larger than the scale $E_{2}=1 /\left(m_{\chi} a^{2}\right)$. This condition $k T\left(z_{\mathrm{dc}}\right) \gg E_{2}$ can be expressed approximately as $m_{\chi} a \gg \sqrt{20}$. 


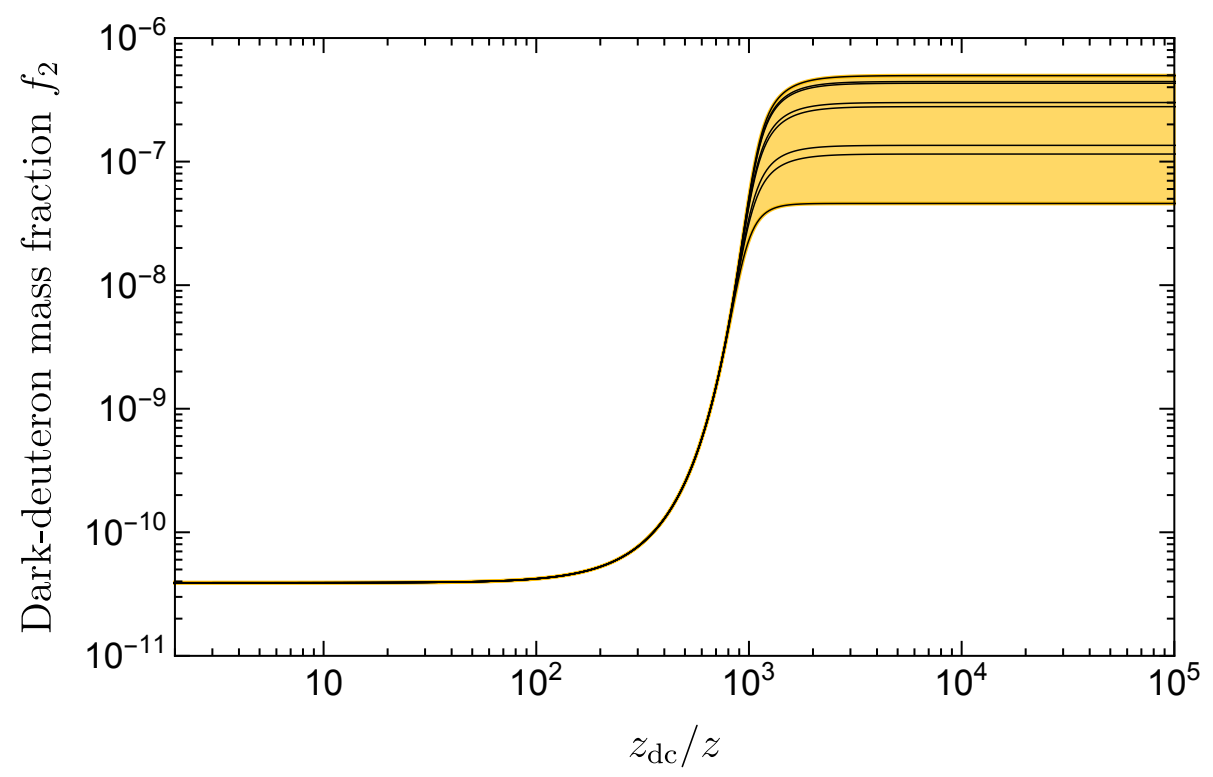

Figure 6. Dark-deuteron mass fraction $f_{2}(z)$ in the early universe as a function of the redshift variable $z_{\mathrm{dc}} / z$ on a $\log$ scale for $m_{\chi}=19 \mathrm{GeV}$ and $a=17 \mathrm{fm}$. The curves are for 8 values of the 3-body parameter: $a_{+} / a=\lambda_{0}^{n / 8}$ with $n=0,1, \cdots, 7$.

\subsection{Numerical results}

Assuming the dark nucleons are identical bosons with a large scattering length, the fewbody parameters are the dark nucleon mass $m_{\chi}$, the scattering length $a$, and the three-body parameter $a_{+}$. For the mass and the scattering length, we use values that can solve smallscale structure problems of the universe. The values that give the best fit to the data points for $\left\langle v \sigma_{\text {elastic }}\right\rangle$ versus $\langle v\rangle$ in figure 1 are $m_{\chi}=19 \mathrm{GeV}$ and $a=17 \mathrm{fm}$. Since the 3-body parameter $a_{+}$is only defined modulo multiplicative factors of $\lambda_{0} \approx 22.69$, the complete range of possibilities is covered by varying $a_{+}$from $a$ to $22.69 a$.

To determine the dark-deuteron mass fraction $f_{2}(z)$ as a function of the redshift $z$, we solve the differential equation in eq. (5.11) subject to the initial condition $f_{2}\left(z_{\mathrm{dc}}\right)=0$. Given the mass $m_{\chi}=19 \mathrm{GeV}$, the decoupling redshift in eq. (5.9) is $z_{\mathrm{dc}} \approx 4 \times 10^{12}$. We want to determine whether a significant fraction $f_{2}$ is ever generated during the subsequent time evolution.

In figure 6 , we show the dark deuteron fraction $f_{2}$ as a function of a red-shift variable $z_{\mathrm{dc}} / z$ on a $\log$ scale. This variable increases from 1 at the decoupling time to infinity at the present time. The band in figure 6 corresponds to minimizing and maximizing $f_{2}$ with respect to $a_{+}$. The individual curves are for eight values of $a_{+}$that are equally spaced on a $\log$ scale between $a$ and $22.69 a$. As $z$ decreases from $z_{\mathrm{dc}}$, the dark deuteron fraction $f_{2}(z)$ increases very quickly to a plateau of about $4 \times 10^{-11}$ from thermal equilibrium between 3 body recombination and dark deuteron breakup. That equilibrium value is consistent with the estimate for the scaling region in eq. (5.13). We could therefore just as well take the initial value of $f_{2}$ at the decoupling red shift to be the equilibrium value given by eq. (5.13). When the dark matter temperature $T$ decreases below the dark deuteron binding energy 


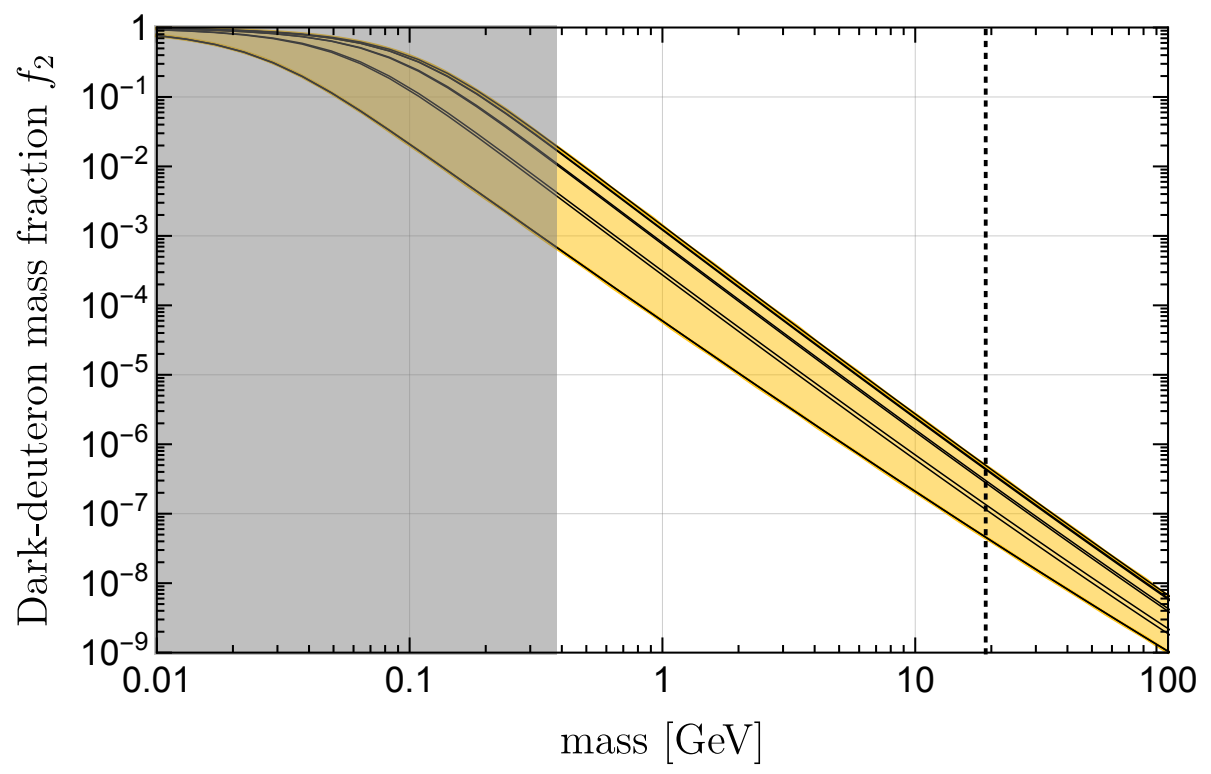

Figure 7. Dark-deuteron mass fraction $f_{2}(z=0)$ at late times as a function of the dark matter mass $m_{\chi}$ with the scattering length $a$ determined by $\sigma_{\text {elastic }} / m_{\chi}=2 \mathrm{~cm}^{2} / \mathrm{g}$. The curves are for 8 values of the 3 -body parameter: $a_{+} / a=\lambda_{0}^{n / 8}$ with $n=0,1, \cdots, 7$. The vertical dotted line marks the mass $m_{\chi}=19 \mathrm{GeV}$ used in figure 6 . The results are reliable only well outside the shaded region where $m_{\chi} a \gg \sqrt{20}$.

$1 /\left(m_{\chi} a^{2}\right)=7.1 \mathrm{keV}$, there is a dramatic increase in $f_{2}$ by 3 or 4 orders of magnitude. This feature is expected from the exponential suppression of the breakup process in eq. (4.6) and from the $z^{4}$ dependence at late times that is predicted by eq. (5.15). The dark-deuteron fraction plateaus at a value $f_{2}(0)$ that depends log-periodically on the 3-body parameter $a_{+}$and can be approximated by

$$
f_{2}(0)=\left(4.6 \times 10^{-8}\right)+\left(6.7 \times 10^{-9}\right) C_{3}\left(a / a_{+}\right)
$$

The maximum value of $f_{2}(0)$ from varying $a_{+}$is larger than the minimum value by a factor of 11. The fraction $f_{2}(0)$ has its minimum when the value of $a_{+} / a$ is just a few percent lower than 1 (or equivalently $\lambda_{0}=22.69$ ), which is the value for which there is total destructive interference in the 3-body recombination rate at zero temperature. It has its maximum when the value of $a_{+} / a$ is just a few percent lower than $\lambda_{0}^{1 / 2}=4.76$.

The results for $f_{2}(z)$ shown in figure 6 are for parameters $m_{\chi}=19 \mathrm{GeV}$ and $a=17 \mathrm{fm}$ that solve small-scale structure problems of the universe, as illustrated in figure 1 . However there are also mechanisms involving baryonic physics that can solve or at least ameliorate the small-scale structure problems. We first relax the constraint on $m_{\chi}$ and $a$ by ignoring the results for $\left\langle v \sigma_{\text {elastic }}\right\rangle$ versus $\langle v\rangle$ from clusters of galaxies. The results in figure 1 from galaxies only are roughly compatible with a cross section that at low velocities approaches $\sigma_{\text {elastic }} / m=2 \mathrm{~cm}^{2} / \mathrm{g}$. This requires the constraint $8 \pi a^{2} / m_{\chi}=2 \mathrm{~cm}^{2} / \mathrm{g}$. Given $m_{\chi}$, the scattering length $a$ is determined. The results for the dark deuteron fraction $f_{2}(0)$ at late times as a function of $m_{\chi}$ are shown in figure 7 . If $m_{\chi}$ is too small, the decoupling temper- 
ature $k T\left(z_{\mathrm{dc}}\right) \approx m_{\chi} / 20$ cannot be much larger than the binding energy $E_{2}=1 /\left(m_{\chi} a^{2}\right)$. In this case, the temperatures after decoupling do not include a scaling region in which $T \gg E_{2}$, so $f_{2}$ is not determined by $a$ and $a_{+}$only, but has additional sensitivity to the range $r_{0}$. This additional sensitivity to $r_{0}$ is avoided if $m_{\chi} a \gg \sqrt{20}$, which implies $m_{\chi} \gg 0.4 \mathrm{GeV}$. This requires $m_{\chi}$ to be well above the shaded region in figure 7 . In the unshaded region, the fraction $f_{2}(0)$ scales roughly as $m_{\chi}^{-2.5}$. At $m_{\chi}=1 \mathrm{GeV}$, the range of $f_{2}(0)$ from varying $a_{+}$is from $6 \times 10^{-5}$ to $2 \times 10^{-3}$. Thus a dark deuteron fraction as large as $10^{-3}$ is possible if the dark-matter elastic cross section at low velocities is $2 \mathrm{~cm}^{2} / \mathrm{g}$.

If the small-scale structure problems of galaxies are ameliorated by mechanisms involving baryonic physics, the constraint on $m_{\chi}$ and $a$ becomes the inequality $8 \pi a^{2} / m_{\chi}<2 \mathrm{~cm}^{2} / \mathrm{g}$. At a given value of $m_{\chi}$, this allows the scattering length to be decreased. This can only decrease the dark deuteron fraction $f_{2}(0)$ at late times.

The results presented above assume the kinetic decoupling temperature $T_{\mathrm{kdc}}$ is very close to the chemical decoupling temperature $T_{\mathrm{dc}}[89]$. We now consider the case when $T_{\mathrm{kdc}}$ is significantly smaller than $T_{\mathrm{dc}}$. The value of $T_{\mathrm{kdc}}$ depends on the interactions between dark matter and ordinary matter. We treat it as an unknown parameter and simply describe how $f_{2}(0)$ scales with $T_{\mathrm{kdc}} / T_{\mathrm{dc}}$. During thermal equilibrium, the dark matter temperature is the same as the photon temperature in eq. $(5.7 \mathrm{~b})$, while after kinetic decoupling the temperature is quadratic in $z$ as in eq. (5.7a). The dark matter temperature can be written as

$$
\begin{array}{rlrl}
T(z) & \approx(1+z) T_{\mathrm{cmb}} & & z>z_{\mathrm{kdc}}, \\
& \approx\left(\frac{1+z}{1+z_{\mathrm{kdc}}}\right)^{2} T_{\mathrm{kdc}} & z<z_{\mathrm{kdc}},
\end{array}
$$

where $z_{\mathrm{kdc}} \approx T_{\mathrm{kdc}} / T_{\mathrm{cmb}}-1$ is the redshift at the kinetic decoupling temperature. By inserting eq. (5.17) into eq. (5.11) instead of eq. (5.8), we obtain the fraction with kinetic decoupling taken into account. Figure 8 shows the fraction $f_{2}(z=0)$ at late times as a function of $T_{\mathrm{kdc}} / T_{\mathrm{dc}}$ for the masses $1 \mathrm{GeV}$ (upper band) and $19 \mathrm{GeV}$ (lower band). As shown in figure 8 , as $T_{\mathrm{kdc}}$ decreases, the fraction decreases, scaling roughly as $\left(T_{\mathrm{kdc}} / T_{\mathrm{dc}}\right)^{1.9}$.

\section{Discussion and conclusion}

The predictions of $\Lambda \mathrm{CDM}$ cosmology face a number of challenges at small scales. These small-scale structure problems can be resolved either by effects of baryons on structure formation or by novel dark matter dynamics. Self-interacting dark matter is a paradigm that can solve the small-scale structure problems in $\Lambda$ CDM cosmology while remaining consistent with other cosmological data. Perhaps the most predictive model of self-interacting dark matter involves a near-threshold S-wave resonance that produces a large scattering length [1]. This near-threshold resonance is a bound state if the scattering length is positive. The signatures for this two-body bound state (darkonium or dark deuteron) in direct detection and directional detection experiments have been studied [2,3]. In this paper, we have studied the production rate of the dark deuteron in the early universe.

We first compared the predictions of the near-threshold S-wave resonance model with cross sections for self-interacting dark matter in different astrophysical objects determined 


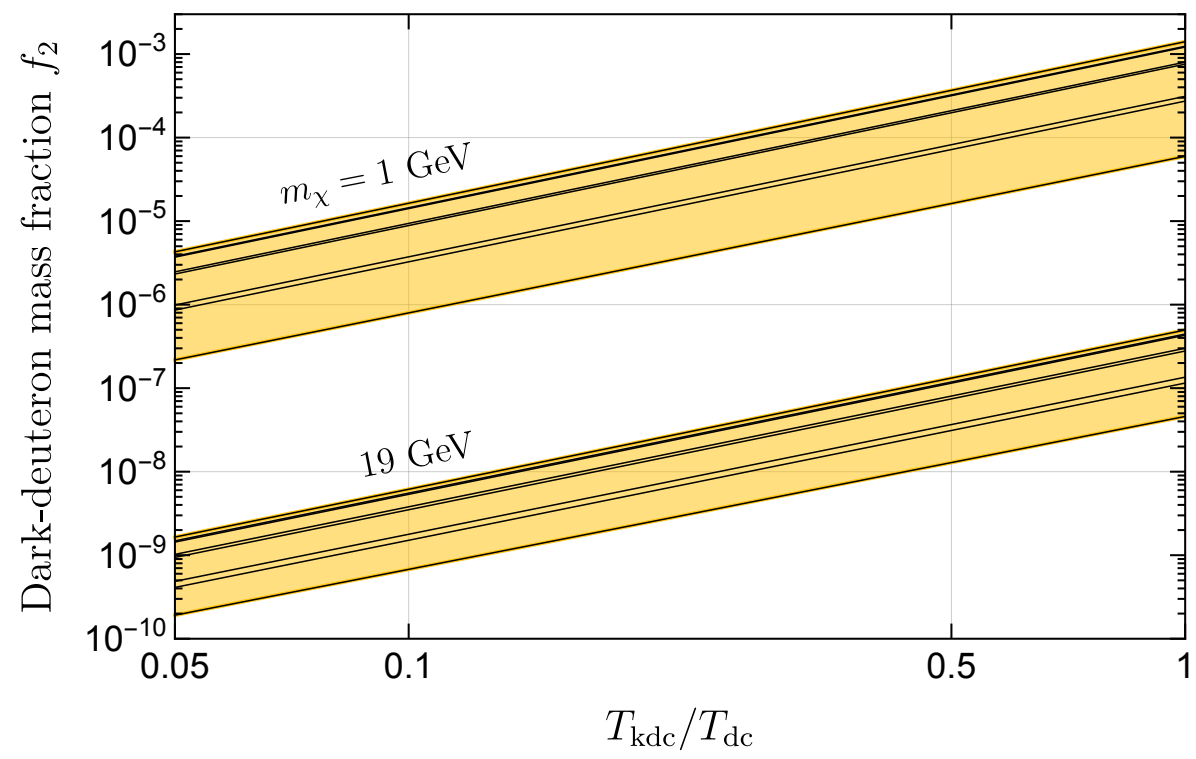

Figure 8. Dark-deuteron mass fraction $f_{2}(z=0)$ at late times as a function of ratio $T_{\mathrm{kdc}} / T_{\mathrm{dc}}$ of the temperatures for kinetic decoupling and chemical decoupling for the masses $1 \mathrm{GeV}$ (upper band) and $19 \mathrm{GeV}$ (lower band). The curves are for 8 values of the 3 -body parameter: $a_{+} / a=\lambda_{0}^{n / 8}$ with $n=0,1, \cdots, 7$.

by Kaplinghat, Tulin and Yu [53]. They showed that a dark-photon model with three adjustable parameters can reproduce the velocity dependence of the self-interaction cross section, which spans two orders of magnitude in velocity [53]. We find that the nearthreshold S-wave resonance model provides an equally good fit to these astrophysical data (see figure 1) with only two adjustable parameters: the mass $m_{\chi}$ of the dark-matter particle and the scattering length $a$. The best-fit values are $m_{\chi}=19 \mathrm{GeV}$ and $a= \pm 17 \mathrm{fm}$.

We have assumed the dark nucleons are identical bosons with a large positive scattering length. The smallest universal bound cluster is the dark deuteron $d_{2}$. The simplest reaction that can form this bound cluster is 3-body recombination into the dark deuteron: $d+d+d \rightarrow$ $d_{2}+d$. The three-body recombination rate is a function of the mass $m_{\chi}$, the scattering length $a$, and a three-body parameter $a_{+}$, with the dependence on $a_{+}$being log-periodic with discrete scaling factor 22.7. If the temperature at decoupling is much larger than the binding energy of the dark deuteron, the present fraction $f_{2}(0)$ of dark matter in the form of dark deuterons is completely determined by these three parameters. For $m_{\chi}=19 \mathrm{GeV}$ and $a=17 \mathrm{fm}$, the fraction $f_{2}(z)$ at early red shifts has an equilibrium value of about $4 \times 10^{-11}$. When the dark-matter temperature decreases to below the binding energy of the dark deuteron, which occurs at a red shift $z \approx 10^{10}, f_{2}(z)$ increases by orders of magnitude to a value between $4 \times 10^{-8}$ and $5 \times 10^{-7}$ that depends on $a_{+}$.

The present fraction $f_{2}(0)$ of dark matter in the form of dark deuterons can be increased by relaxing the constraint on $m_{\chi}$ and $a$ from solving small-scale structure problems and decreasing $m_{\chi}$. However the decoupling temperature must be much larger than the binding energy of the dark deuteron for $f_{2}(0)$ to be insensitive to the range of the interactions. 
Given this constraint, $f_{2}(0)$ cannot be larger than about $10^{-3}$. If the system remains in thermal equilibrium longer after chemical decoupling, the fraction $f_{2}(0)$ decreases, scaling approximately as the 2 nd power of the ratio of the temperatures for kinetic and chemical decoupling. We conclude that a significant population of dark deuterons cannot be produced in the early universe by 3 -body recombination of dark matter particles with a large scattering length. Since the production of dark deuterons is a bottleneck for the formation of larger bound clusters, we conclude that the formation of bound clusters in the early universe would require additional microphysics. An example is a light mediator that allows radiative fusion reactions.

If the large scattering length $a$ is negative, the smallest universal bound clusters are Efimov clusters $d_{3}$ ("dark tritons"). The simplest reaction that can form bound clusters is 4 -body recombination into a dark triton: $d+d+d+d \rightarrow d_{3}+d$. The rate for 4 -body recombination is suppressed compared to the rate for 3-body recombination by an additional factor of the number density of dark matter particles. Since a significant population of dark deuterons cannot be produced in the early universe by 3-body recombination, a significant population of dark tritons cannot be produced by 4-body recombination either. Since the production of dark tritons is a bottleneck for the production of larger dark nuclei, a significant number of dark nuclei will not be formed in the early universe if the dark nucleons are identical bosons with a large negative scattering length.

Identical bosons are not the only types of particles for which there is dramatic enhancement of the 3-body recombination rate at low temperature when the scattering length is large. The degree to which 3-body recombination is enhanced depends on the symmetries and mass ratios of the particles with large scattering lengths. Three-body recombination requires the three particles to come within a distance of order the de Broglie wavelength of the final-state particles, which is of order $1 / a$ if the collision energy is small. For identical bosons, the 3-body recombination rate $K_{3}(T)$ in the low-temperature limit is proportional to $a^{4}$. If the dark matter consists of the two spin states of a spin- $\frac{1}{2}$ fermion, $K_{3}(T \rightarrow 0)$ is suppressed by $\left(r_{0} / a\right)^{2}$, where $r_{0}$ is the range, because the Pauli exclusion principle suppresses the contribution from the region where the separations of the three fermions are all of order $a$. If the dark matter consists of the four spin states of two spin- $\frac{1}{2}$ fermions, there is no such suppression and $K_{3}(T \rightarrow 0)$ is proportional to $a^{4}$.

We can also show that a significant fraction of dark deuterons cannot form once the dark matter particles fall inside the gravitational potential well of a galaxy. It is easy to put an upper bound on the rate of increase in the dark deuteron fraction in the Milky Way from 3 -body recombination. The maximum possible rate of increase in $n_{2}$ is $\left(67.1 a^{4} / m_{\chi}\right) n_{1}^{3}$. The dark matter mass density in the solar system, which is about $8 \mathrm{kpc}$ from the center of the Milky Way, is $m_{\chi} n_{1}=0.3 \mathrm{GeV} / \mathrm{cm}^{3}$. If feedback between strongly interacting dark matter and baryons is taken into account, the radius of the dark matter core of the Milky Way may be about $0.3 \mathrm{kpc}[90]$. The dark matter mass density in the core of the Milky Way may be about $m_{\chi} n_{1}=8 \mathrm{GeV} / \mathrm{cm}^{3}$. For $m_{\chi}=19 \mathrm{GeV}$ and $a=17 \mathrm{fm}$, the maximum rate of increase of $f_{2}$ is about $10^{-51} / \mathrm{s}$. The age of the Milky Way is about $10 \mathrm{Gyr} \approx 3 \times 10^{17} \mathrm{~s}$, so we see that the dark deuteron fraction remains negligible. If we relax the constraints on $m_{\chi}$ and $a$ from solving small-scale structure problems but keep the binding energy of the 
dark deuteron small compared to the decoupling temperature, the rate of increase of $f_{2}$ can be made larger at most by about an order of magnitude. Dwarf galaxies can have higher dark matter densities than the Milky Way, but the rate of increase in the dark deuteron fraction is small in those systems too.

Although 3-body recombination of dark matter particles is unable to build up a large fraction of dark deuterons in the early universe, it may still have a significant effect on dark matter annihilation. If a pair of dark matter particles has an annihilation scattering channel, the constituents of a dark deuteron will eventually annihilate once the dark deuteron is formed. Three-body recombination therefore provides an additional annihilation channel. If the dark matter particles have a large scattering length $a$, the annihilation scattering cross section and the dark deuteron decay rate are both determined by $m_{\chi}$ and $a$ up to a multiplicative constant that cancels in their ratio. The resonant enhancement of annihilation scattering can induce a second period of dark matter annihilation after the thermal freezeout [91]. The effects of reannihilation have been explored in a dark photon model [91]. A near-threshold S-wave resonance model provides a more predictive framework in which the effects of reannihilation through 3-body recombination can also be easily explored.

\section{Acknowledgments}

We thank M. Kaplinghat, S. Tulin, and H.-B. Yu for providing their data in figure 1 and M. Kaplinghat for valuable comments. This research of E.B. was supported in part by the Department of Energy under the grant DE-SC0011726 and by the National Science Foundation under grant PHY-1607190. D.K. is supported by NSFC through Grant No. 11875112. R.L. is supported by German Research Foundation (DFG) under Grant Nos. EXC-1098, KO 4820/1-1, FOR 2239, and from the European Research Council (ERC) under the European Union's Horizon 2020 research and innovation programme (grant agreement No. 637506, " $\nu$ Directions") awarded to Joachim Koop. We acknowledge the INT program "Multi-Scale Problems Using Effective Field Theories (INT-18-1b)", during which this work was finalized. D.K. would like to thank the hospitality of High Energy Theory Group, Ohio State University, where an important part of this work was carried out.

Open Access. This article is distributed under the terms of the Creative Commons Attribution License (CC-BY 4.0), which permits any use, distribution and reproduction in any medium, provided the original author(s) and source are credited.

\section{References}

[1] E. Braaten and H.W. Hammer, Universal Two-body Physics in Dark Matter near an S-wave Resonance, Phys. Rev. D 88 (2013) 063511 [arXiv: 1303.4682] [InSPIRE].

[2] R. Laha and E. Braaten, Direct detection of dark matter in universal bound states, Phys. Rev. D 89 (2014) 103510 [arXiv:1311.6386] [INSPIRE].

[3] R. Laha, Directional detection of dark matter in universal bound states, Phys. Rev. D 92 (2015) 083509 [arXiv: 1505.02772] [INSPIRE]. 
[4] W. Shepherd, T.M.P. Tait and G. Zaharijas, Bound states of weakly interacting dark matter, Phys. Rev. D 79 (2009) 055022 [arXiv:0901.2125] [InSPIRE].

[5] M. Yu. Khlopov, A.G. Mayorov and E. Yu. Soldatov, Composite Dark Matter and Puzzles of Dark Matter Searches, Int. J. Mod. Phys. D 19 (2010) 1385 [arXiv:1003.1144] [inSPIRE].

[6] J.M. Cline, Z. Liu, G. Moore and W. Xue, Composite strongly interacting dark matter, Phys. Rev. D 90 (2014) 015023 [arXiv:1312.3325] [INSPIRE].

[7] R. Foot, Mirror dark matter: Cosmology, galaxy structure and direct detection, Int. J. Mod. Phys. A 29 (2014) 1430013 [arXiv:1401.3965] [InSPIRE].

[8] K. Petraki, L. Pearce and A. Kusenko, Self-interacting asymmetric dark matter coupled to a light massive dark photon, JCAP 07 (2014) 039 [arXiv: 1403.1077] [INSPIRE].

[9] W. Detmold, M. McCullough and A. Pochinsky, Dark Nuclei I: Cosmology and Indirect Detection, Phys. Rev. D 90 (2014) 115013 [arXiv:1406.2276] [InSPIRE].

[10] W. Detmold, M. McCullough and A. Pochinsky, Dark nuclei. II. Nuclear spectroscopy in two-color QCD, Phys. Rev. D 90 (2014) 114506 [arXiv:1406.4116] [INSPIRE].

[11] M.B. Wise and Y. Zhang, Stable Bound States of Asymmetric Dark Matter, Phys. Rev. D 90 (2014) 055030 [Erratum ibid. D 91 (2015) 039907] [arXiv: 1407.4121] [inSPIRE].

[12] B. von Harling and K. Petraki, Bound-state formation for thermal relic dark matter and unitarity, JCAP 12 (2014) 033 [arXiv:1407.7874] [InSPIRE].

[13] M.B. Wise and Y. Zhang, Yukawa Bound States of a Large Number of Fermions, JHEP 02 (2015) 023 [Erratum ibid. 10 (2015) 165] [arXiv:1411.1772] [INSPIRE].

[14] E. Hardy, R. Lasenby, J. March-Russell and S.M. West, Big Bang Synthesis of Nuclear Dark Matter, JHEP 06 (2015) 011 [arXiv:1411.3739] [INSPIRE].

[15] T. Appelquist et al., Stealth Dark Matter: Dark scalar baryons through the Higgs portal, Phys. Rev. D 92 (2015) 075030 [arXiv:1503.04203] [INSPIRE].

[16] E. Hardy, R. Lasenby, J. March-Russell and S.M. West, Signatures of Large Composite Dark Matter States, JHEP 07 (2015) 133 [arXiv: 1504.05419] [INSPIRE].

[17] K. Petraki, M. Postma and M. Wiechers, Dark-matter bound states from Feynman diagrams, JHEP 06 (2015) 128 [arXiv: 1505.00109] [INSPIRE].

[18] H. An, B. Echenard, M. Pospelov and Y. Zhang, Probing the Dark Sector with Dark Matter Bound States, Phys. Rev. Lett. 116 (2016) 151801 [arXiv:1510.05020] [INSPIRE].

[19] Y. Tsai, L.-T. Wang and Y. Zhao, Dark Matter Annihilation Decay at The LHC, Phys. Rev. D 93 (2016) 035024 [arXiv: 1511.07433] [INSPIRE].

[20] X.-J. Bi, Z. Kang, P. Ko, J. Li and T. Li, Asymmetric Dark Matter Bound State, Phys. Rev. D 95 (2017) 043540 [arXiv: 1602.08816] [INSPIRE].

[21] G.D. Kribs and E.T. Neil, Review of strongly-coupled composite dark matter models and lattice simulations, Int. J. Mod. Phys. A 31 (2016) 1643004 [arXiv:1604.04627] [InSPIRE].

[22] C. Kouvaris, K. Langæble and N.G. Nielsen, The Spectrum of Darkonium in the Sun, JCAP 10 (2016) 012 [arXiv: 1607.00374] [INSPIRE].

[23] F. Nozzoli, A balance for Dark Matter bound states, Astropart. Phys. 91 (2017) 22 [arXiv: 1608.00405] [INSPIRE]. 
[24] A. Butcher, R. Kirk, J. Monroe and S.M. West, Can Tonne-Scale Direct Detection Experiments Discover Nuclear Dark Matter?, JCAP 10 (2017) 035 [arXiv: 1610.01840] [INSPIRE].

[25] P. Asadi, M. Baumgart, P.J. Fitzpatrick, E. Krupczak and T.R. Slatyer, Capture and Decay of Electroweak WIMPonium, JCAP 02 (2017) 005 [arXiv: 1610.07617] [INSPIRE].

[26] K. Petraki, M. Postma and J. de Vries, Radiative bound-state-formation cross-sections for dark matter interacting via a Yukawa potential, JHEP 04 (2017) 077 [arXiv:1611.01394] [INSPIRE].

[27] M. Cirelli, P. Panci, K. Petraki, F. Sala and M. Taoso, Dark Matter's secret liaisons: phenomenology of a dark $\mathrm{U}(1)$ sector with bound states, JCAP 05 (2017) 036 [arXiv: 1612.07295] [INSPIRE].

[28] S.J. Lonsdale, M. Schroor and R.R. Volkas, Asymmetric Dark Matter and the hadronic spectra of hidden QCD, Phys. Rev. D 96 (2017) 055027 [arXiv: 1704.05213] [INSPIRE].

[29] M.I. Gresham, H.K. Lou and K.M. Zurek, Nuclear Structure of Bound States of Asymmetric Dark Matter, Phys. Rev. D 96 (2017) 096012 [arXiv:1707.02313] [INSPIRE].

[30] A. Mitridate, M. Redi, J. Smirnov and A. Strumia, Dark Matter as a weakly coupled Dark Baryon, JHEP 10 (2017) 210 [arXiv:1707.05380] [INSPIRE].

[31] G. Elor, H. Liu, T.R. Slatyer and Y. Soreq, Complementarity for Dark Sector Bound States, Phys. Rev. D 98 (2018) 036015 [arXiv: 1801.07723] [INSPIRE].

[32] J. Harz and K. Petraki, Radiative bound-state formation in unbroken perturbative non-Abelian theories and implications for dark matter, JHEP 07 (2018) 096 [arXiv: 1805.01200] [INSPIRE].

[33] S. Biondini and M. Laine, Thermal dark matter co-annihilating with a strongly interacting scalar, JHEP 04 (2018) 072 [arXiv: 1801.05821] [INSPIRE].

[34] S. Biondini, Bound-state effects for dark matter with Higgs-like mediators, JHEP 06 (2018) 104 [arXiv: 1805.00353] [INSPIRE].

[35] R.H. Cyburt, B.D. Fields, K.A. Olive and T.-H. Yeh, Big Bang Nucleosynthesis: 2015, Rev. Mod. Phys. 88 (2016) 015004 [arXiv: 1505.01076] [InSPIRE].

[36] R. Consiglio, P.F. de Salas, G. Mangano, G. Miele, S. Pastor and O. Pisanti, PArthENoPE reloaded, Comput. Phys. Commun. 233 (2018) 237 [arXiv:1712.04378] [INSPIRE].

[37] G. Krnjaic and K. Sigurdson, Big Bang Darkleosynthesis, Phys. Lett. B 751 (2015) 464 [arXiv: 1406.1171] [INSPIRE].

[38] E. Braaten and H.W. Hammer, Universality in few-body systems with large scattering length, Phys. Rept. 428 (2006) 259 [cond-mat/0410417] [INSPIRE].

[39] D.H. Weinberg, J.S. Bullock, F. Governato, R. Kuzio de Naray and A.H.G. Peter, Cold dark matter: controversies on small scales, Proc. Nat. Acad. Sci. 112 (2015) 12249 [arXiv: 1306.0913] [INSPIRE].

[40] A. Brooks, Re-Examining Astrophysical Constraints on the Dark Matter Model, Annalen Phys. 526 (2014) 294 [arXiv:1407.7544] [INSPIRE].

[41] A. Pontzen and F. Governato, Cold dark matter heats up, Nature 506 (2014) 171 [arXiv: 1402.1764] [INSPIRE]. 
[42] A. Del Popolo and M. Le Delliou, Small scale problems of the $\Lambda$ CDM model: a short review, Galaxies 5 (2017) 17 [arXiv:1606.07790] [InSPIRE].

[43] J.S. Bullock and M. Boylan-Kolchin, Small-Scale Challenges to the $\Lambda$ CDM Paradigm, Ann. Rev. Astron. Astrophys. 55 (2017) 343 [arXiv:1707.04256] [INSPIRE].

[44] M.R. Buckley and A.H.G. Peter, Gravitational probes of dark matter physics, arXiv: 1712.06615 [INSPIRE].

[45] D.N. Spergel and P.J. Steinhardt, Observational evidence for selfinteracting cold dark matter, Phys. Rev. Lett. 84 (2000) 3760 [astro-ph/9909386] [INSPIRE].

[46] S. Tulin and H.-B. Yu, Dark Matter Self-interactions and Small Scale Structure, Phys. Rept. 730 (2018) 1 [arXiv: 1705. 02358] [INSPIRE].

[47] R. Laha, Effect of hydrodynamical-simulation-inspired dark matter velocity profile on directional detection of dark matter, Phys. Rev. D 97 (2018) 043004 [arXiv:1610.08632] [INSPIRE].

[48] C. Chin, R. Grimm, P. Julienne and E. Tiesinga, Feshbach resonances in ultracold gases, Rev. Mod. Phys. 82 (2010) 1225 [arXiv:0812.1496].

[49] F. Luo, G.C. McBane, G. Kim, C.F. Giese and W.R. Gentry, The weakest bond: Experimental observation of helium dimer, J. Chem. Phys. 98 (1993) 3564.

[50] M. Rocha et al., Cosmological Simulations with Self-Interacting Dark Matter I: Constant Density Cores and Substructure, Mon. Not. Roy. Astron. Soc. 430 (2013) 81 [arXiv: 1208.3025] [INSPIRE].

[51] A.H.G. Peter, M. Rocha, J.S. Bullock and M. Kaplinghat, Cosmological Simulations with Self-Interacting Dark Matter II: Halo Shapes vs. Observations, Mon. Not. Roy. Astron. Soc. 430 (2013) 105 [arXiv: 1208.3026] [INSPIRE].

[52] O.D. Elbert, J.S. Bullock, M. Kaplinghat, S. Garrison-Kimmel, A.S. Graus and M. Rocha, A Testable Conspiracy: Simulating Baryonic Effects on Self-Interacting Dark Matter Halos, Astrophys. J. 853 (2018) 109 [arXiv:1609.08626] [INSPIRE].

[53] M. Kaplinghat, S. Tulin and H.-B. Yu, Dark Matter Halos as Particle Colliders: Unified Solution to Small-Scale Structure Puzzles from Dwarfs to Clusters, Phys. Rev. Lett. 116 (2016) 041302 [arXiv: 1508.03339] [INSPIRE].

[54] A.B. Newman, T. Treu, R.S. Ellis and D.J. Sand, The Density Profiles of Massive, Relaxed Galaxy Clusters: II. Separating Luminous and Dark Matter in Cluster Cores, Astrophys. J. 765 (2013) 25 [arXiv:1209.1392] [INSPIRE].

[55] A.B. Newman et al., The Density Profiles of Massive, Relaxed Galaxy Clusters: I. The Total Density Over 3 Decades in Radius, Astrophys. J. 765 (2013) 24 [arXiv:1209.1391] [INSPIRE].

[56] S.-H. Oh, W.J.G. de Blok, E. Brinks, F. Walter and R.C. Kennicutt Jr., Dark and luminous matter in THINGS dwarf galaxies, Astron. J. 141 (2011) 193 [arXiv:1011. 0899] [INSPIRE].

[57] R. Kuzio de Naray, S.S. McGaugh and W.J.G. de Blok, Mass Models for Low Surface Brightness Galaxies with High Resolution Optical Velocity Fields, Astrophys. J. 676 (2008) 920 [arXiv:0712.0860] [INSPIRE].

[58] E. Braaten, E. Johnson and H. Zhang, Zero-range effective field theory for resonant wino dark matter. Part I. Framework, JHEP 11 (2017) 108 [arXiv:1706. 02253] [InSPIRE]. 
[59] E. Braaten, E. Johnson and H. Zhang, Zero-range effective field theory for resonant wino dark matter. Part II. Coulomb resummation, JHEP 02 (2018) 150 [arXiv:1708. 07155] [INSPIRE].

[60] E. Braaten, E. Johnson and H. Zhang, Zero-range effective field theory for resonant wino dark matter. Part III. Annihilation effects, JHEP 05 (2018) 062 [arXiv:1712.07142] [INSPIRE].

[61] D. Clowe et al., A direct empirical proof of the existence of dark matter, Astrophys. J. 648 (2006) L109 [astro-ph/0608407] [INSPIRE].

[62] V. Springel and G. Farrar, The Speed of the bullet in the merging galaxy cluster 1E0657-56, Mon. Not. Roy. Astron. Soc. 380 (2007) 911 [astro-ph/0703232] [INSPIRE].

[63] J. Lee and E. Komatsu, Bullet Cluster: A Challenge to LCDM Cosmology, Astrophys. J. 718 (2010) 60 [arXiv: 1003.0939] [inSPIRE].

[64] F. Kahlhoefer, K. Schmidt-Hoberg, M.T. Frandsen and S. Sarkar, Colliding clusters and dark matter self-interactions, Mon. Not. Roy. Astron. Soc. 437 (2014) 2865 [arXiv:1308.3419] [INSPIRE].

[65] C. Lage and G. Farrar, Constrained Simulation of the Bullet Cluster, Astrophys. J. 787 (2014) 144 [arXiv: 1312.0959] [INSPIRE].

[66] C. Lage and G.R. Farrar, The Bullet Cluster is not a Cosmological Anomaly, JCAP 02 (2015) 038 [arXiv:1406.6703] [INSPIRE].

[67] D. Kraljic and S. Sarkar, How rare is the Bullet Cluster (in a $\Lambda$ CDM universe)?, JCAP 04 (2015) 050 [arXiv: 1412.7719] [INSPIRE].

[68] R. Massey et al., Dark matter dynamics in Abell 3827: new data consistent with standard cold dark matter, Mon. Not. Roy. Astron. Soc. 477 (2018) 669 [arXiv:1708.04245] [INSPIRE].

[69] D. Harvey, R. Massey, T. Kitching, A. Taylor and E. Tittley, The non-gravitational interactions of dark matter in colliding galaxy clusters, Science 347 (2015) 1462 [arXiv: 1503.07675] [INSPIRE].

[70] A. Robertson, R. Massey and V. Eke, What does the Bullet Cluster tell us about self-interacting dark matter?, Mon. Not. Roy. Astron. Soc. 465 (2017) 569 [arXiv: 1605.04307] [INSPIRE].

[71] J.I. Read, M.G. Walker and P. Steger, The case for a cold dark matter cusp in Draco, Mon. Not. Roy. Astron. Soc. 481 (2018) 860 [arXiv: 1805.06934] [InSPIRE].

[72] S.Y. Kim, A.H.G. Peter and D. Wittman, In the Wake of Dark Giants: New Signatures of Dark Matter Self Interactions in Equal Mass Mergers of Galaxy Clusters, Mon. Not. Roy. Astron. Soc. 469 (2017) 1414 [arXiv: 1608.08630] [INSPIRE].

[73] V. Efimov, Energy levels arising form the resonant two-body forces in a three-body system, Phys. Lett. B 33 (1970) 563 [inSPIRE].

[74] V. Efimov, Energy levels of three resonantly interacting particles, Nucl. Phys. A 210 (1973) 157 [INSPIRE].

[75] A.O. Gogolin, C. Mora and R. Egger, Analytical Solution of the Bosonic Three-Body Problem, Phys. Rev. Lett. 100 (2008) 140404 [arXiv:0802.0549].

[76] W. Schollkopf and J.P. Toennies, Nondestructive Mass Selection of Small van der Waals Clusters, Science 266 (1994) 1345.

[77] J. Voigtsberger et al., Imaging the structure of the trimer systems ${ }^{4} \mathrm{He}_{3}$ and ${ }^{3} \mathrm{He}^{4} \mathrm{He}_{2}, \mathrm{Nat}$. Commun. 5 (2014) 5765. 
[78] T. Kraemer et al., Evidence for Efimov quantum states in an ultracold gas of caesium atoms, Nature 440 (2006) 315 [cond-mat/0512394].

[79] E. Braaten, H.W. Hammer, D. Kang and L. Platter, Three-Body Recombination of Identical Bosons with a Large Positive Scattering Length at Nonzero Temperature, Phys. Rev. A 78 (2008) 043605 [arXiv:0801.1732] [INSPIRE].

[80] J. Macek, S.Y. Ovchinnikov and G. Gasaneo, Exact solution for three particles interacting via zero-range potentials, Phys. Rev. A 73 (2006) 032704.

[81] L. Platter, H.W. Hammer and U.-G. Meissner, The Four boson system with short range interactions, Phys. Rev. A 70 (2004) 052101 [cond-mat/0404313] [InSPIRE].

[82] H.W. Hammer and L. Platter, Universal Properties of the Four-Body System with Large Scattering Length, Eur. Phys. J. A 32 (2007) 113 [nucl-th/0610105] [INSPIRE].

[83] J. von Stecher, J.P. D'Incao and C.H. Greene, Four-body legacy of the Efimov effect, Nat. Phys. 5 (2009) 417 [arXiv:0810.3876].

[84] F. Ferlaino et al., Evidence for Universal Four-Body States Tied to an Efimov Trimer, Phys. Rev. Lett. 102 (2009) 140401 [arXiv:0903.1276].

[85] J. von Stecher, Universal Five- and Six-Body Droplets Tied to an Efimov Trimer, Phys. Rev. Lett. 107 (2011) 200402 [arXiv:1106.2319] [INSPIRE].

[86] Particle Data Group collaboration, C. Patrignani et al., Review of Particle Physics, Chin. Phys. C 40 (2016) 100001 [InSPIRE].

[87] C. Armendariz-Picon and J.T. Neelakanta, How Cold is Cold Dark Matter?, JCAP 03 (2014) 049 [arXiv: 1309.6971] [INSPIRE].

[88] G. Steigman, B. Dasgupta and J.F. Beacom, Precise Relic WIMP Abundance and its Impact on Searches for Dark Matter Annihilation, Phys. Rev. D 86 (2012) 023506 [arXiv: 1204.3622] [INSPIRE].

[89] T. Binder, T. Bringmann, M. Gustafsson and A. Hryczuk, Early kinetic decoupling of dark matter: when the standard way of calculating the thermal relic density fails, Phys. Rev. D 96 (2017) 115010 [arXiv:1706. 07433] [INSPIRE].

[90] M. Kaplinghat, R.E. Keeley, T. Linden and H.-B. Yu, Tying Dark Matter to Baryons with Self-interactions, Phys. Rev. Lett. 113 (2014) 021302 [arXiv:1311.6524] [INSPIRE].

[91] T. Binder, M. Gustafsson, A. Kamada, S.M.R. Sandner and M. Wiesner, Reannihilation of self-interacting dark matter, Phys. Rev. D 97 (2018) 123004 [arXiv:1712.01246] [INSPIRE]. 